\title{
SINGULARITIES ON THE 2-DIMENSIONAL MODULI SPACES OF STABLE SHEAVES ON K3 SURFACES
}

\author{
NOBUAKI ONISHI AND KŌTA YOSHIOKA
}

\section{INTRODUCTION}

Let $X$ be a K3 surface over $\mathbb{C}$. Mukai introduced a lattice structure $\langle\quad, \quad\rangle$ on $H^{*}(X, \mathbb{Z}):=\bigoplus_{i} H^{2 i}(X, \mathbb{Z})$ by

$$
\begin{aligned}
\langle x, y\rangle & :=-\int_{X} x^{\vee} \wedge y \\
& =\int_{X}\left(x_{1} \wedge y_{1}-x_{0} \wedge y_{2}-x_{2} \wedge y_{0}\right),
\end{aligned}
$$

where $x_{i} \in H^{2 i}(X, \mathbb{Z})\left(\right.$ resp. $\left.y_{i} \in H^{2 i}(X, \mathbb{Z})\right)$ is the $2 i$-th component of $x$ (resp. $\left.y\right)$ and $x^{\vee}=x_{0}-x_{1}+x_{2}$. It is now called the Mukai lattice. For a coherent sheaf $E$ on $X$, we can attach an element of $H^{*}(X, \mathbb{Z})$ called the Mukai vector

$$
v(E):=\operatorname{ch}(E) \sqrt{\operatorname{td}_{X}}=\operatorname{ch}(E)\left(1+\rho_{X}\right),
$$

where $\operatorname{ch}(E)$ is the Chern character of $E, \operatorname{td}_{X}$ the Todd class of $X$ and $\rho_{X}$ the fundamental cohomology class of $X\left(\int_{X} \rho_{X}=1\right)$.

Definition 0.1. [Y3] We fix an ample divisor $H$ on $X$ and an element $G \in K(X) \otimes \mathbb{Q}$ with $\operatorname{rk} G>0$.

(i) Let $E$ be a torsion free sheaf on $X$. $E$ is $G$-twisted semi-stable (resp. stable) with respect to $H$, if

$$
\frac{\chi(G, F(n H))}{\operatorname{rk}(F)} \leq \frac{\chi(G, E(n H))}{\operatorname{rk}(E)}, n \gg 0
$$

for $0 \subsetneq F \subsetneq E$ (resp. the inequality is strict).

(ii) For a $w \in H^{*}(X, \mathbb{Q})_{\text {alg }}:=\mathbb{Q} \oplus \operatorname{NS}(X) \otimes \mathbb{Q} \oplus \mathbb{Q} \rho_{X}$ with $\operatorname{rk} w>0$, we define the $w$-twisted semi-stability as the $G$-twisted semi-stability, where $G \in K(X) \otimes \mathbb{Q}$ satisfies $v(G)=w$.

Matsuki and Wentworth $[\mathrm{M}-\mathrm{W}$ constructed the moduli space of $w$-twisted semi-stable sheaves $E$ with $v(E)=v$. We denote it by $\bar{M}_{H}^{w}(v)$. If $w=v\left(\mathcal{O}_{X}\right)$, then the $v\left(\mathcal{O}_{X}\right)$-twisted semi-stability is nothing but the usual Gieseker's semi-stability. Hence we denote $\bar{M}_{H}^{v\left(\mathcal{O}_{X}\right)}(v)$ by $\bar{M}_{H}(v)$.

Assume that $v$ is an isotropic Mukai vector. In [A] , Abe considered the singularities of $\bar{M}_{H}(v)$. Replacing $\bar{M}_{H}(v)$ by $\bar{M}_{H}^{v}(v)$, we shall generalize Abe's results:

Theorem 0.1. (1) $\bar{M}_{H}^{v}(v)$ is normal.

(2) For a suitable choice of $\alpha$ with $\left|\left\langle\alpha^{2}\right\rangle\right| \ll 1$, there is a surjective morphism $\phi_{\alpha}: \bar{M}_{H}^{v+\alpha}(v)=$ $M_{H}^{v+\alpha}(v) \rightarrow \bar{M}_{H}^{v}(v)$ which becomes a minimal resolution of the singularities.

(3) Let $x$ be a point of $\bar{M}_{H}^{v}(v)$ corresponding to the $S$-equivalence class $\bigoplus_{i=0}^{n} E_{i}^{\oplus a_{i}}$, where $E_{i}, 0 \leq i \leq n$ are $v$-twisted stable sheaves. Then the matrix $\left(-\left\langle v\left(E_{i}\right), v\left(E_{j}\right)\right\rangle\right)_{i, j=0}^{n}$ is of affine type $\tilde{A}_{n}, \tilde{D}_{n}, \tilde{E}_{n}$. Assume that $a_{0}=1$. Then the singularity of $\bar{M}_{H}^{v}(v)$ at $x$ is a rational double point of type $A_{n}, D_{n}, E_{n}$ according as the type of the matrix $\left(-\left\langle v\left(E_{i}\right), v\left(E_{j}\right)\right\rangle\right)_{i, j=1}^{n}$.

Moreover we shall show that the Weyl chamber of the corresponding finite Lie algebra appears as a parameter space of $\alpha$.

If the matrix is of type $\tilde{A}_{n}$, then the assertion (1) is due to Abe [A, Thm. 3.3]. Moreover if $n=1,2$, then he showed the assertion (3). The assertion (2) is also contained in [A, Thm. 3.3]. The main point of the proof is due to Matsuki and Wentworth [M-W] or Ellingsrud and Göttsche [E-G]. The $v$-twisted stability naturally appears in the study of the Fourier-Mukai transforms. In [Y3], [Y5], we studied the FourierMukai transform and showed that the Fourier-Mukai transform preserves the $v$-twisted semi-stability under suitable assumptions. So the $v$-twisted semi-stability is important and this is our original motivation to study the moduli space of $v$-twisted semi-stable sheaves. Another motivation is the following: For the GIT quotients related to the moduli spaces of vector bundles on curves with additional structures, the wall crossing behaviors have been studied by several authors. In particular, Thaddeus [1] described the wall crossing behavior as a sequence of blowing-ups and blowing-downs and used it to show the Verlinde formula. 
For the rank two case, Ellingsrud and Göttsche [E-G] studied the similar variation problem for the moduli space of stable sheaves on a K3 surface. In this case, Mukai's elementary transformation appears. For all these examples, the exceptional locus of the blowing-up is irreducible. So it is interesting to construct an example with a reducible exceptional lucus, and a rational double point will be a simple and interesting example to consider.

Our main idea to study the exceptional locus is the same as the one in Y1 to study the Brill-Noether locus of sheaves on K3 surfaces. Let us explain the contents of this note. In section 1, we show that the Donaldson's determinant line bundle on $\bar{M}_{H}^{v}(v)$ is ample. The $w$-twisted stability depends on the choice of $w$. Hence we introduce a suitable parameter space of $w$ and introduce a chamber structure on this parameter space. This chamber will become a Weyl chamber of a finite simple Lie algebra in the next section. By using a special kind of Fourier-Mukai transform called reflection, we compare the twisted stability for two $w$ (Proposition 1.12). Section 2 is the main part of this note. We first describe the exceptional locus of the resolution as a Brill-Noether locus of sheaves, under the assumption that $w=v+\alpha,\left|\left\langle\alpha^{2}\right\rangle\right| \ll 1$ belongs to a special chamber. By using the Weyl group action on the parameter space, we can give a set-theoretic description of the exceptional locus for general cases. Finally we shall prove that $\bar{M}_{H}^{v}(v)$ is normal. In section 3, we give some examples of singular moduli spaces by using the surjectivity of the period map.

\section{Definitions}

Let $L$ be a lattice (or a $\mathbb{Q}$-vector space with a bilinear form) with a weight 2 Hodge structure: $L \otimes \mathbb{C}=$ $\bigoplus_{p+q=2} L^{p, q}$. We set $L_{a l g}:=L \cap L^{1,1}$. The Mukai lattice $H^{*}(X, \mathbb{Z})$ has a Hodge structure:

$$
\begin{aligned}
& H^{2,0}\left(H^{*}(X, \mathbb{C})\right)=H^{2,0}(X) \\
& H^{1,1}\left(H^{*}(X, \mathbb{C})\right)=H^{0,0}(X) \oplus H^{1,1}(X) \oplus H^{2,2}(X), \\
& H^{0,2}\left(H^{*}(X, \mathbb{C})\right)=H^{0,2}(X)
\end{aligned}
$$

Then $H^{*}(X, \mathbb{Z})_{\text {alg }}=\mathbb{Z} \oplus \operatorname{Pic}(X) \oplus \mathbb{Z} \rho_{X}$.

1.1. Twisted stability. Let $G$ be an element of $K(X) \otimes \mathbb{Q}$ with $\operatorname{rk} G>0$. We fix an ample divisor $H$ on $X$. For a coherent sheaf $E$ on $X$, we define the $G$-twisted rank, degree, and Euler characteristic of $E$ by

$$
\begin{aligned}
\operatorname{rk}_{G}(E) & :=\operatorname{rk}\left(G^{\vee} \otimes E\right), \\
\operatorname{deg}_{G}(E) & :=\left(c_{1}\left(G^{\vee} \otimes E\right), H\right), \\
\chi_{G}(E) & :=\chi\left(G^{\vee} \otimes E\right) .
\end{aligned}
$$

We shall rewrite the condition (0.3) on the twisted stability. By the Riemann-Roch theorem, we get that

$$
\begin{aligned}
\frac{\chi(G, E(n H))}{\operatorname{rk}(G) \operatorname{rk}(E)}-\frac{\chi(G, F(n H))}{\operatorname{rk}(G) \operatorname{rk}(F)}= & n\left(\frac{\operatorname{deg}_{G}(E)}{\operatorname{rk}_{G}(E)}-\frac{\operatorname{deg}_{G}(F)}{\operatorname{rk}_{G}(F)}\right)+\left(\frac{\chi_{G}(E)}{\operatorname{rk}_{G}(E)}-\frac{\chi_{G}(F)}{\operatorname{rk}_{G}(F)}\right) \\
= & n\left(\frac{\left(c_{1}(E), H\right)}{\operatorname{rk}(E)}-\frac{\left(c_{1}(F), H\right)}{\operatorname{rk}(F)}\right)+\left(\frac{\chi(E)}{\operatorname{rk}(E)}-\frac{\chi(F)}{\operatorname{rk}(F)}\right) \\
& +\left(\frac{c_{1}(E)}{\operatorname{rk}(E)}-\frac{c_{1}(F)}{\operatorname{rk}(F)}, \frac{c_{1}(G)}{\operatorname{rk} G}\right) .
\end{aligned}
$$

Let $\varphi: \operatorname{Pic}(X) \otimes \mathbb{Q} \rightarrow H^{\perp}$ be the orthogonal projection. Then the twisted stability depends only on $\varphi\left(c_{1}(G) / \operatorname{rk} G\right) \in H^{\perp}$ and it is nothing but the twisted stability due to Matsuki-Wentworth $\mathrm{M}-\mathrm{W}$.

Definition 1.1. A polarization $H$ is general with respect to $v$, if the following condition holds:

(*) for every $\mu$-semi-stable sheaf $E$ with $v(E)=v$, if $F \subset E$ satisfies $\left(c_{1}(F), H\right) / \operatorname{rk} F=\left(c_{1}(E), H\right) / \operatorname{rk} E$, then $c_{1}(F) / \mathrm{rk} F=c_{1}(E) / \mathrm{rk} E$.

If $H$ is general with respect to $v$, then the $w$-twisted semi-stability does not depend on the choice of $w$. The following theorem was proved in $\mathrm{M}-\mathrm{W}]$.

Theorem 1.1. $\mathbb{M - W}$ Let $w$ be an element of $H^{*}(X, \mathbb{Q})_{\text {alg }}$ such that $\mathrm{rk} w>0$. Then there is a coarse moduli scheme $\bar{M}_{H}^{w}(v)$ of $S$-equivalence classes of $w$-twisted semi-stable sheaves $E$ with $v(E)=v . \bar{M}_{H}^{w}(v)$ is a projective scheme.

Definition 1.2. We denote the open subscheme of $\bar{M}_{H}^{w}(v)$ consisting of $w$-twisted stable sheaves by $M_{H}^{w}(v)$.

If $w=v\left(\mathcal{O}_{X}\right)$, then we denote $\bar{M}_{H}^{w}(v)\left(\operatorname{resp} . M_{H}^{w}(v)\right)$ by $\bar{M}_{H}(v)\left(\right.$ resp. $\left.M_{H}(v)\right)$.

Proposition 1.2. $\bar{M}_{H}^{w}(v) \neq \emptyset$ if $\left\langle v^{2}\right\rangle \geq-2$.

Proof. We may assume that $v$ is primitive. If $H$ is general with respect to $v$, then [Y2, Thm. 8.1] implies that $\bar{M}_{H}(v) \neq \emptyset$. By the study of the chamber structure (cf. [Y33, sect. 1, Prop. 4.2]), we get our claim 
1.2. Line bundles on $\bar{M}_{H}^{w}(v)$. Throughout this note, $v:=r+\xi+a \rho_{X}, \xi \in \operatorname{Pic}(X)$ is a primitive isotropic Mukai vector with $r>0$.

We define a homomorphism which preserves the Hodge structure and the metric:

$$
\begin{array}{clc}
\delta: H^{2}(X, \mathbb{Q}) & \rightarrow \quad H^{*}(X, \mathbb{Q}) \\
D & \mapsto D+\frac{(D, \xi)}{r} \rho_{X} .
\end{array}
$$

We denote $\delta(D)$ by $\widehat{D}$. Then we have an orthogonal decomposition:

$$
H^{*}(X, \mathbb{Q})=\left(\mathbb{Q} v \oplus \mathbb{Q} \rho_{X}\right) \perp \delta\left(H^{2}(X, \mathbb{Q})\right) .
$$

Let $\theta_{v}^{\alpha}: v^{\perp} \rightarrow H^{2}\left(M_{H}^{v+\alpha}(v), \mathbb{Z}\right)$ be the Mukai homomorphism defined by

$$
\theta_{v}^{\alpha}(x):=\frac{1}{\rho}\left[p_{M_{H}^{v+\alpha}(v) *}\left((\operatorname{ch} \mathcal{E}) \sqrt{\operatorname{td}_{X}} x^{\vee}\right)\right]_{1},
$$

where $\mathcal{E}$ is a quasi-universal family of similitude $\rho$. If $x \in\left(v^{\perp} / \mathbb{Z} v\right) \otimes \mathbb{Q}$, then we have a $\mathbb{Q}$-line bundle $L(x)$ on $M_{H}^{v+\alpha}(v)$ such that $c_{1}(L(x))=\theta_{v}^{\alpha}(x)$. For $\widehat{H}=H+\{(H, \xi) / r\} \rho_{X}, L(r \widehat{H})$ is the Donaldson's determinant line bundle and $\mathrm{J}$. Li [ [i] showed that canonically $L(r \widehat{H})$ extends to a line bundle on $\bar{M}_{H}^{v+\alpha}(v)$. We also denote this extension by $L(r \widehat{H})$. Then $L(r \widehat{H})$ is a nef and big line bundle and we have a contraction map from the Gieseker moduli space to the Uhlenbeck moduli space. Hence $L(r \widehat{H})$ is important.

One of the reason we consider the $v$-twisted stability is the following proposition.

Proposition 1.3. $L(r \widehat{H})$ is an ample line bundle on $\bar{M}_{H}^{v}(v)$.

Proof. We recall the construction of $\bar{M}_{H}^{v}(v)$ in Y4. Let $E$ be a $v$-twisted stable sheaf with $v(E)=v$. We set $N:=\chi(E, E(n H))$. Let $Q:=\operatorname{Quot}_{E(-n H) \oplus N}^{v} / X / \mathbb{C}$ be a quot-scheme parametrizing all quotients $E(-n H)^{\oplus N} \rightarrow F$ such that $v(F)=v$ and $\mathcal{O}_{Q}^{\oplus N} \otimes E(-n H) \rightarrow \mathcal{Q}$ the universal quotient. Let $Q^{s s}$ be an open subscheme of $Q$ consisting of $q \in Q$ such that

(i) $\mathcal{Q}_{q}$ is $v$-twisted semi-stable,

(ii) $\operatorname{Hom}\left(E, E^{\oplus N}\right) \rightarrow \operatorname{Hom}\left(E, \mathcal{Q}_{q}(n H)\right)$ is an isomorphism,

(iii) $\operatorname{Ext}^{i}\left(E, \mathcal{Q}_{q}(n H)\right)=0, i>0$.

Then we have an isomorphism $\mathcal{O}_{Q^{s s}}^{\oplus N} \rightarrow p_{Q^{s s_{*}}}\left(\mathcal{Q} \otimes p_{X}^{*}(E(-n H))^{\vee}\right)$. $Q$ has a natural action of $G L(N)$. We set $\mathcal{L}_{m}:=\operatorname{det} p_{Q !}\left(\mathcal{Q} \otimes p_{X}^{*}(E(-m H))^{\vee}\right)$. Since $\mathcal{Q}$ is $G L(N)$-linearized, $\mathcal{L}_{m}$ is also $G L(N)$-linearized. By the construction of $Q, \mathcal{L}_{n+m}, m \gg 0$ gives an embedding of $Q$ to a Grassmann variety. Thus $\mathcal{L}_{n+m}$, $m \gg 0$ is ample. Let $T:=\operatorname{det}\left(\mathcal{O}_{Q}^{\oplus N}\right)$ be the $G L(N)$-linearized line bundle induced by the standard action of $G L(N)$ on $\mathcal{O}_{Q}^{\oplus N}$. The center $\mathbb{C}^{\times} \subset G L(N)$ acts trivially on $Q$ and the action on $\mathcal{L}_{m}$ is the multiplication by $\chi\left(E, E\left(\mathcal{O}_{X}(-m H)\right)\right)$-th power of constant. By a simple calculation, we see that $\chi\left(E, E\left(\mathcal{O}_{X}(-m H)\right)\right)=$ $r^{2} m^{2}\left(H^{2}\right) / 2$. Hence $\mathcal{L}:=\mathcal{L}_{n+m}^{\otimes m^{2}} \otimes \mathcal{L}_{n}^{\otimes-(n+m)^{2}}$ and $\mathcal{L}^{\prime}:=\mathcal{L}_{n+m}^{\otimes m^{2}} \otimes T^{\otimes-(n+m)^{2}}$ have $P G L(N)$-linearizations. By the construction of the moduli space, $\bar{M}_{H}^{v}(v)$ is described as a GIT quotient $Q^{s s} \rightarrow \bar{M}_{H}^{v}(v)$, where $n \gg 0$ and $\mathcal{L}^{\prime}$ is the linearization. Since $\mathcal{L}_{\mid Q^{s s}}=\mathcal{L}_{\mid Q^{s s}}^{\prime}$ as $P G L(N)$-line bundles, $\mathcal{L}_{\mid Q^{s s}}$ descends to an ample line bundle on $\bar{M}_{H}^{v}(v)$. We note that

$$
\mathcal{L}=\mathcal{L}_{n+m}^{\otimes m^{2}} \otimes \mathcal{L}_{n}^{\otimes-(n+m)^{2}}=\operatorname{det} p_{Q !}\left(\mathcal{Q} \otimes p_{X}^{*}(L)^{\vee}\right),
$$

where $L=m^{2} E(-(n+m) H)-(n+m)^{2} E(-n H) \in K(X)$. Since $\operatorname{det} p_{Q^{s s !}}\left(\mathcal{Q} \otimes p_{X}^{*}(E)^{\vee}\right)=\mathcal{O}_{Q^{s s}}$,

$$
\operatorname{det} p_{Q^{s s !}}\left(\mathcal{Q} \otimes p_{X}^{*}(L)^{\vee}\right)=\operatorname{det} p_{Q^{s s !}}\left(\mathcal{Q} \otimes p_{X}^{*}\left(L^{\prime}\right)^{\vee}\right),
$$

where $L^{\prime}=L-\left(m^{2}-(n+m)^{2}\right) E$. Since $v\left(L^{\prime}\right)=r m n(m+n) \widehat{H}$, we get our claim.

Corollary 1.4. (1) If $\xi \in \mathbb{Q} H$, then $\bar{M}_{H}(v)=\bar{M}_{H}^{v}(v)$. Hence $L(r \widehat{H})$ is an ample line bundle on $\bar{M}_{H}(v)$.

(2) Let $\mathcal{M}_{n}$ (resp. $\overline{\mathcal{M}}_{n}$ ) be the moduli space of polarized (resp. quasi-polarized) K3 surfaces $(X, H)$ with $\left(H^{2}\right)=2 n$. We set $v:=r+d H+a \rho_{X}, d^{2}\left(H^{2}\right)=2 r a$. Then we have a morphism of the moduli spaces $\mathcal{M}_{n} \rightarrow \overline{\mathcal{M}}_{n^{\prime}}:(X, H) \mapsto\left(\bar{M}_{H}(v), L(\widehat{H})\right)$ where $n=r a / d^{2}$ and $n^{\prime}$ is determined by the primitive class in $\mathbb{Q} L(\widehat{H}) \cap \operatorname{Pic}\left(\bar{M}_{H}(v)\right)$.

In particular, if $v:=r+H+a \rho_{X}$ satisfies $\operatorname{gcd}(r, a)=1$, then $M_{H}(v)$ is compact and $\widehat{H}=H+2 a \rho_{X}$ gives a canonical primitive polarization of $M_{H}(v)=M_{H}^{v}(v)$.

Remark 1.1. If $\operatorname{gcd}(r, d)=1$, then $M_{H}(v)$ consists of $\mu$-stable locally free sheaves for a general $X$. For a special $X, M_{H}(v)$ may consist of properly $\mu$-semi-stable sheaves. Indeed let $X \rightarrow \mathbb{P}^{1}$ be an elliptic K3 surface with a section $\sigma$. We set $H:=\sigma+3 f$, where $f$ is a fiber of $\pi$. If $\operatorname{Pic}(X)=\mathbb{Z} \sigma \oplus \mathbb{Z} f$, then $H$ is an 
ample divisor with $\left(H^{2}\right)=4$. We set $v=2+H+\rho_{X}$. Then $\left\langle v^{2}\right\rangle=0$ and every member of $M_{H}(v)$ is given by

$$
E:=\operatorname{ker}\left(\mathcal{O}_{X}(\sigma+f) \oplus \mathcal{O}_{X}(2 f) \stackrel{e v}{\rightarrow} \mathbb{C}_{s}\right),
$$

$s \in X$.

For the Mukai homomorphism, Mukai [Mu2] showed the following.

Theorem 1.5 (Mukai). Assume that $M_{H}^{v+\alpha}(v)$ is compact. Then $\theta_{v}^{\alpha}$ is surjective and the kernel is $\mathbb{Z} v$. Moreover $\theta_{v}^{\alpha}: v^{\perp} / \mathbb{Z} v \rightarrow H^{2}\left(M_{H}^{v+\alpha}(v), \mathbb{Z}\right)$ is a Hodge isometry.

By (1.4) and (1.5), we have a sequence of Hodge isometries:

$$
H^{2}(X, \mathbb{Q}) \rightarrow \delta\left(H^{2}(X, \mathbb{Q})\right) \rightarrow\left(v^{\perp} / \mathbb{Z} v\right) \otimes_{\mathbb{Z}} \mathbb{Q} \rightarrow H^{2}\left(M_{H}^{v+\alpha}(v), \mathbb{Q}\right) .
$$

Then since $\widehat{H}=\delta(H) \in v^{\perp}$, we have an isometry

$$
\delta\left(H^{\perp}\right)_{\text {alg }} \rightarrow\left(\left(\widehat{H}^{\perp} \cap v^{\perp}\right) / \mathbb{Z} v\right)_{a l g} \otimes \mathbb{Q} .
$$

In particular $\left(\left(\widehat{H}^{\perp} \cap v^{\perp}\right) / \mathbb{Z} v\right)_{a l g}$ is negative definite.

1.3. Chamber structure. We shall study the dependence of $\bar{M}_{H}^{w}(v)$ on $w$. By (1.3), we may assume that $w=v+\alpha, \alpha \in \delta\left(H^{\perp}\right)_{a l g}$. Let $u$ be a Mukai vector such that $0<\operatorname{rk} u<\operatorname{rk} v,\langle v, u\rangle \leq 0,\left\langle u^{2}\right\rangle=-2$ and $\langle u, \widehat{H}\rangle=0$. We define a wall $W_{u} \subset \delta\left(H^{\perp}\right)_{\text {alg }} \otimes_{\mathbb{Q}} \mathbb{R}$ with respect to $v$ by

$$
W_{u}:=\left\{\alpha \in \delta\left(H^{\perp}\right)_{a l g} \otimes \mathbb{R} \mid\langle v+\alpha, u\rangle=0\right\} .
$$

For a properly $v+\alpha$-twisted semi-stable sheaf $E$ with $v(E)=v$, we consider the Jordan-Hölder filtration

$$
0 \subset F_{1} \subset F_{2} \subset \cdots \subset F_{s}=E
$$

of $E$ with respect to the $v+\alpha$-twisted stability, that is, $E_{i}:=F_{i} / F_{i-1}$ is a $v+\alpha$-twisted stable sheaf with

$$
\begin{aligned}
& \left(c_{1}\left(E_{i}\right), H\right) / \operatorname{rk} E_{i}=\left(c_{1}(E), H\right) / \operatorname{rk} E=(\xi, H) / r, \\
& \left\langle v+\alpha, v\left(E_{i}\right)\right\rangle / \operatorname{rk} E_{i}=\langle v+\alpha, v\rangle / \operatorname{rk} E .
\end{aligned}
$$

We set $u_{i}:=v\left(E_{i}\right)$. Then we see that $\left\langle u_{i}, \widehat{H}\right\rangle=\left(c_{1}\left(E_{i}\right), H\right)-(\xi, H) r_{i} / r=0$ and $\left\langle v+\alpha, u_{i}\right\rangle=0$.

Lemma 1.6. $\left\langle v, u_{i}\right\rangle \leq 0$ and $\left\langle u_{i}^{2}\right\rangle=-2$ for some $i$.

Proof. Since $u_{i} / \mathrm{rk} u_{i}-v / \mathrm{rk} v \in \widehat{H}^{\perp} \cap \rho_{X}^{\perp}$ and $\left(\widehat{H}^{\perp} \cap \rho_{X}^{\perp}\right)_{\text {alg }}$ is negative semi-definite, $\left\langle\left(u_{i} / \mathrm{rk} u_{i}-v / \mathrm{rk} v\right)^{2}\right\rangle \leq$ 0 . Then $\left\langle u_{i}^{2}\right\rangle \leq 2\left\langle u_{i}, v\right\rangle\left(\operatorname{rk} u_{i} / \operatorname{rk} v\right)$. Since $\sum_{j}\left\langle u_{j}, v\right\rangle=\langle v, v\rangle=0$, we get $\left\langle u_{i}, v\right\rangle \leq 0$ for some $i$. In particular $\left\langle u_{i}^{2}\right\rangle<0$ provided that $\left\langle u_{i}, v\right\rangle<0$. If $\left\langle u_{i}, v\right\rangle=0$, then $u_{i} \in \widehat{H}^{\perp} \cap v^{\perp}$. Since $\operatorname{rk} u_{i}<\operatorname{rk} v$, we get $u_{i} \notin \mathbb{Z} v$. Then (1.11) implies that $\left\langle u_{i}^{2}\right\rangle<0$. Since $\left\langle u_{i}^{2}\right\rangle \geq-2$, we conclude that $\left\langle u_{i}^{2}\right\rangle=-2$.

Therefore $\alpha \in W_{u_{i}}$. We set

$$
\mathcal{U}:=\left\{\begin{array}{l|l}
u \in H^{*}(X, \mathbb{Z})_{a l g} \mid \begin{array}{l}
\left\langle u^{2}\right\rangle=-2,\langle v, u\rangle \leq 0,\langle\widehat{H}, u\rangle=0, \\
0<\operatorname{rk} u<\operatorname{rk} v
\end{array}
\end{array} .\right.
$$

For a fixed $v$ and $H, \mathcal{U}$ is a finite set.

Lemma 1.7. If $\alpha$ does not lie on any wall $W_{u}, u \in \mathcal{U}$, then $\bar{M}_{H}^{v+\alpha}(v)=M_{H}^{v+\alpha}(v)$. In particular, $\bar{M}_{H}^{v+\alpha}(v)$ is a K3 surface.

Definition 1.3. Let $\mathcal{C}$ be a connected component of $\delta\left(H^{\perp}\right)_{\text {alg }} \otimes_{\mathbb{Q}} \mathbb{R} \backslash \cup_{u \in \mathcal{U}} W_{u}$. We call $\mathcal{C}$ a chamber.

As is proved in M-W, we get

Proposition 1.8. The $v+\alpha$-twisted stability does not depend on the choice of $\alpha \in \mathcal{C}$. If $\beta$ belongs to the closure of $\mathcal{C}$, then we have a morphism $\bar{M}_{H}^{v+\alpha}(v) \rightarrow \bar{M}_{H}^{v+\beta}(v)$ for $\alpha \in \mathcal{C}$. In particular, we have a morphism $\phi_{\alpha}: \bar{M}_{H}^{v+\alpha}(v) \rightarrow \bar{M}_{H}^{v}(v)$ for $\left|\left\langle\alpha^{2}\right\rangle\right| \ll 1$.

Let $T \subset v^{\perp}$ be a sufficiently small neighborhood of 0 . Then $W_{u}$ intersects $T$ if and only if $\langle v, u\rangle=0$. Since we are interested in the neighborhood of $v$, we may assume that the defining equation of a wall $W_{u}$ belongs to the subset

$$
\mathcal{U}^{\prime}:=\{u \in \mathcal{U} \mid\langle v, u\rangle=0\} .
$$

By the same argument as above, we get the following.

Lemma 1.9. Let $E$ be a properly $v$-twisted semi-stable sheaf with $v(E)=v$ and

$$
0 \subset F_{1} \subset F_{2} \subset \cdots \subset F_{s}=E
$$

the Jordan-Hölder filtration of $E$ with respect to the $v$-twisted stability. Then $\left\langle v\left(F_{i} / F_{i-1}\right)^{2}\right\rangle=-2$. 
1.4. Reflection. For an $\alpha \in \delta\left(H^{\perp}\right)_{\text {alg }}$ with $\left|\left\langle\alpha^{2}\right\rangle\right| \ll 1$, let $F$ be a $v+\alpha$-twisted stable torsion free sheaf such that

(i) $\left\langle v(F)^{2}\right\rangle=-2$,

(ii) $\langle v(F), \widehat{H}\rangle / \mathrm{rk} F=\left(c_{1}(F), H\right) / \mathrm{rk} F-(\xi, H) / r=0$ and

(iii) $\langle v, v(F)\rangle=\langle\alpha, v(F)\rangle=0$.

By (i), $F$ is a rigid torsion free sheaf, and hence $F$ is locally free.

Let $\mathcal{E}$ be a coherent sheaf on $X \times X$ which is defined by an exact sequence

$$
0 \rightarrow \mathcal{E} \rightarrow p_{1}^{*}\left(F^{\vee}\right) \otimes p_{2}^{*}(F) \stackrel{e v}{\rightarrow} \mathcal{O}_{\Delta} \rightarrow 0,
$$

where $p_{i}: X \times X \rightarrow X, i=1,2$ are projections. We consider the Fourier-Mukai transform induced by $\mathcal{E}$ :

$$
\begin{array}{ccc}
\mathcal{F}_{\mathcal{E}}: \quad \mathbf{D}(X) & \rightarrow & \mathbf{D}(X) \\
x & \mapsto \mathbf{R} p_{2 *}\left(p_{1}^{*}(x) \otimes \mathcal{E}\right),
\end{array}
$$

where $\mathbf{D}(X)$ is the bounded derived category of $X$. Up to shift, the inverse of $\mathcal{F}_{\mathcal{E}}$ is given by

$$
\begin{array}{ccc}
\widehat{\mathcal{F}}_{\mathcal{E}}: \quad \mathbf{D}(X) & \rightarrow & \mathbf{D}(X) \\
y & \mapsto & \mathbf{R} \operatorname{Hom}_{p_{1}}\left(\mathcal{E}, p_{2}^{*}(y)\right) .
\end{array}
$$

Definition 1.4. Let $E$ be a coherent sheaf on $X$.

(i) We denote the $i$-th cohomology sheaf of $\mathcal{F}_{\mathcal{E}}(E)\left(\right.$ resp. $\left.\widehat{\mathcal{F}}_{\mathcal{E}}(E)\right)$ by $\mathcal{F}_{\mathcal{E}}^{i}(E)\left(\right.$ resp. $\left.\widehat{\mathcal{F}}_{\mathcal{E}}^{i}(E)\right)$.

(ii) $E$ satisfies $\mathrm{WIT}_{i}$ with respect to $\mathcal{F}_{\mathcal{E}}\left(\right.$ resp. $\widehat{\mathcal{F}}_{\mathcal{E}}$ ), if $\mathcal{F}_{\mathcal{E}}^{j}(E)=0$ (resp. $\widehat{\mathcal{F}}_{\mathcal{E}}^{j}(E)=0$ ) for $j \neq i$.

The Fourier-Mukai transform $\mathcal{F}_{\mathcal{E}}$ induces an isometry of the Mukai lattice $\mathcal{F}_{\mathcal{E}}: H^{*}(X, \mathbb{Z}) \rightarrow H^{*}(X, \mathbb{Z})$. Let $R_{v(F)}: H^{*}(X, \mathbb{Z}) \rightarrow H^{*}(X, \mathbb{Z})$ be the reflection defined by the (-2)-vector $v(F)$ :

$$
R_{v(F)}(u)=u+\langle u, v(F)\rangle v(F), u \in H^{*}(X, \mathbb{Z}) .
$$

Then we see that $\mathcal{F}_{\mathcal{E}}=-R_{v(F)}$. Thus the Fourier-Mukai transform $\mathcal{F}_{\mathcal{E}}$ is the geometric realization of the reflection $R_{v(F)}$.

Lemma 1.10. Let $G$ be a $v+\alpha$-twisted semi-stable sheaf such that $\operatorname{deg}(G) / \operatorname{rk} G=\operatorname{deg}(F) / \operatorname{rk} F$ and $\chi(E+A, G) \geq 0$, where $E, A \in K(X) \otimes \mathbb{Q}$ satisfy $v(E)=v, v(A)=\alpha$. Then

$$
\operatorname{Ext}^{2}\left(\mathcal{E}_{\mid\{x\} \times X}, G\right)=0
$$

for all $x \in X$.

Proof. Assume that there is a non-zero homomorphism $\varphi: G \rightarrow \mathcal{E}_{\mid\{x\} \times X}$. Then we have a non-zero homomorphism $\psi: G \rightarrow F$. Since $G$ is a $v+\alpha$-twisted semi-stable sheaf with $\operatorname{deg}(G) / \operatorname{rk} G=\operatorname{deg}(F) / \operatorname{rk} F$ and $F$ is $v+\alpha$-twisted stable, we get that $0 \leq \chi(E+A, G) / \operatorname{rk} G \leq \chi(E+A, F) / \operatorname{rk} F=0$. Hence $\chi(E+A, G)=0$ and $\psi$ is surjective. Thus $\operatorname{im} \varphi$ contains $F$. On the other hand, by the construction of $\mathcal{E}_{\mid\{x\} \times X}, \mathcal{E}_{\mid\{x\} \times X}$ does not contain $F$. Therefore $\operatorname{Hom}\left(G, \mathcal{E}_{\mid\{x\} \times X}\right)=0$. By the Serre duality, we get $\operatorname{Ext}^{2}\left(\mathcal{E}_{\mid\{x\} \times X}, G\right)=0$.

Lemma 1.11. Let $E, A \in K(X) \otimes \mathbb{Q}$ be as in Lemma 1.10. Let $G$ be a $v+\alpha$-twisted semi-stable sheaf such that $\operatorname{deg}(G) / \operatorname{rk} G=\operatorname{deg}(F) / \operatorname{rk} F$ and $\chi(E+A, G)=0$. Then the evaluation map $\phi: \operatorname{Hom}(F, G) \otimes F \rightarrow G$ is injective and coker $\phi$ is a $v+\alpha$-twisted semi-stable sheaf.

Proof. By the $v+\alpha$-twisted semi-stability of $E$ and $F$, we see that $\operatorname{deg}(\operatorname{im} \phi) / \operatorname{rk}(\operatorname{im} \phi)=\operatorname{deg}(F) / \operatorname{rk} F$ and $\chi(E+A, \operatorname{im} \phi)=0$. Hence we get $\operatorname{deg}(\operatorname{ker} \phi) / \operatorname{rk}(\operatorname{ker} \phi)=\operatorname{deg}(F) / \operatorname{rk} F$ and $\chi(E+A, \operatorname{ker} \phi)=0$. Assume that $\operatorname{ker} \phi \neq 0$. By the $v+\alpha$-twisted semi-stability of $\operatorname{Hom}(F, G) \otimes F$, $\operatorname{ker} \phi$ is $v+\alpha$-twisted semi-stable. Then we see that $\operatorname{ker} \phi \cong F^{\oplus k}$, which implies that $\operatorname{Hom}(F, \operatorname{ker} \phi) \neq 0$. On the other hand, $\phi$ induces an isomorphism $\operatorname{Hom}(F, G) \otimes \operatorname{Hom}(F, F) \rightarrow \operatorname{Hom}(F, G)$. Hence we have $\operatorname{Hom}(F, \operatorname{ker} \phi)=0$, which is a contradiction. Therefore $\operatorname{ker} \phi=0$.

Proposition 1.12. We set $\alpha^{ \pm}:= \pm \epsilon v(F)+\alpha$, where $0<\epsilon \ll 1$.

(1) Let $E$ be a $v+\alpha^{-}$-twisted semi-stable sheaf with $v(E)=v$. Then $\mathrm{WIT}_{1}$ holds for $E$ with respect to $\mathcal{F}_{\mathcal{E}}$ and $\mathcal{F}_{\mathcal{E}}^{1}(E)$ is a $v+\alpha^{+}$-twisted semi-stable sheaf.

(2) Conversely, for a $v+\alpha^{+}$-twisted semi-stable sheaf $E$ with $v(E)=v, \mathrm{WIT}_{1}$ holds with respect to $\widehat{\mathcal{F}}_{\mathcal{E}}$ and $\widehat{\mathcal{F}}_{\mathcal{E}}^{1}(E)$ is a $v+\alpha^{-}$-twisted semi-stable sheaf.

(3) Moreover $\mathcal{F}_{\mathcal{E}}$ preserves the $S$-equivalence classes. Hence we have an isomorphism

$$
\bar{M}_{H}^{v+\alpha^{-}}(v) \rightarrow \bar{M}_{H}^{v+\alpha^{+}}(v) .
$$


Proof. We take an element $A \in K(X) \otimes \mathbb{Q}$ such that $v(A)=\alpha$. We note that $F$ is $(v \pm \epsilon v(F)+\alpha)$-twisted stable for $0 \leq \epsilon \ll 1$. We first prove (1). We note that $E$ is $v+\alpha^{-}$-twisted semi-stable. By the definition of $\mathcal{E}$, we get an exact sequence

$$
\begin{array}{r}
0 \longrightarrow p_{2 *}\left(\mathcal{E} \otimes p_{1}^{*}(E)\right) \longrightarrow \operatorname{Hom}^{1}(F, E) \otimes F \longrightarrow p_{2 *}\left(\mathcal{E} \otimes p_{1}^{*}(E)\right) \longrightarrow \operatorname{Ext}^{1}(F, E) \otimes F \longrightarrow \operatorname{Ext}^{2}(F, E) \otimes F \longrightarrow 0 \\
\longrightarrow R^{2} p_{2 *}\left(\mathcal{E} \otimes p_{1}^{*}(E)\right) \longrightarrow \operatorname{Ex} \longrightarrow 0
\end{array}
$$

Since $\operatorname{deg} F / \operatorname{rk} F=\operatorname{deg} E / \operatorname{rk} E$ and $\chi(E-\epsilon F+A, F) / \operatorname{rk} F=-2 \epsilon / \operatorname{rk} F<0=\chi(E-\epsilon F+A, E) / \mathrm{rk} E$, the $v+\alpha^{-}$-twisted semi-stability of $E$ and $F$ imply that $\operatorname{Ext}^{2}(F, E)=\operatorname{Hom}(E, F)^{\vee}=0$. Thus $R^{2} p_{2 *}\left(\mathcal{E} \otimes p_{1}^{*}(E)\right)=$ 0 . Since $E$ is $v+\alpha$-twisted semi-stable, Lemma 1.11 implies that $\operatorname{Hom}(F, E) \otimes F \rightarrow E$ is injective, and hence $p_{2 *}\left(\mathcal{E} \otimes p_{1}^{*}(E)\right)=0$. Therefore $\mathrm{WIT}_{1}$ holds for $E$ and $\mathcal{F}_{\mathcal{E}}^{1}(E)$ is a $v+\alpha$-twisted semi-stable sheaf with $v\left(\mathcal{F}_{\mathcal{E}}^{1}(E)\right)=R_{v(F)}(v)=v$. Assume that $\mathcal{F}_{\mathcal{E}}^{1}(E)$ is not $v+\alpha^{+}$-twisted semi-stable. Then there is an exact sequence

$$
0 \rightarrow G_{1} \rightarrow \mathcal{F}_{\mathcal{E}}^{1}(E) \rightarrow G_{2} \rightarrow 0
$$

such that $G_{1}$ is a $v+\alpha$-twisted semi-stable sheaf with $\operatorname{deg}_{E}\left(G_{1}\right)=\chi\left(E+A, G_{1}\right)=0$ and $G_{2}$ is a $v+\alpha^{+}$-twisted stable sheaf with $\chi\left(E+\epsilon F+A, G_{2}\right)<0$. By Lemma 1.10, we get $\widehat{\mathcal{F}}_{\mathcal{E}}^{2}\left(G_{1}\right)=0$. Since $\chi\left(E+\epsilon F+A, G_{2}\right)<0$ and $\chi(E+\epsilon F+A, F)=2 \epsilon>0, \widehat{\mathcal{F}}_{\mathcal{E}}^{0}\left(G_{2}\right)=\operatorname{Hom}\left(F, G_{2}\right) \otimes F=0$. Therefore $\mathrm{WIT}_{1}$ holds for $G_{1}, G_{2}$ and we get an exact sequence

$$
0 \rightarrow \widehat{\mathcal{F}}_{\mathcal{E}}^{1}\left(G_{1}\right) \rightarrow E \rightarrow \widehat{\mathcal{F}}_{\mathcal{E}}^{1}\left(G_{2}\right) \rightarrow 0 .
$$

Since $\chi\left(F, \widehat{\mathcal{F}}_{\mathcal{E}}^{1}\left(G_{2}\right)\right)=-\chi\left(F, G_{2}\right)>0$, we get a contradiction.

(2) Conversely, let $E$ be a $v+\alpha^{+}$-twisted semi-stable sheaf with $v(E)=v$. Then we have an exact sequence

$$
\begin{aligned}
0 \longrightarrow \operatorname{Hom}_{p_{1}}\left(\mathcal{O}_{\Delta}, p_{2}^{*}(E)\right) \longrightarrow \operatorname{Hom}(F, E) \otimes F \longrightarrow \operatorname{Hom}_{p_{1}}\left(\mathcal{E}, p_{2}^{*}(E)\right) \\
\longrightarrow \operatorname{Ext}_{p_{1}}^{1}\left(\mathcal{O}_{\Delta}, p_{2}^{*}(E)\right) \longrightarrow \operatorname{Ext}_{p_{1}}^{1}\left(\mathcal{E}, p_{2}^{*}(E)\right) \\
\longrightarrow \operatorname{Ext}_{p_{1}}^{2}\left(\mathcal{O}_{\Delta}, p_{2}^{*}(E)\right) \longrightarrow F \longrightarrow \operatorname{Ext}^{2}(F, E) \otimes F \longrightarrow \operatorname{Ext}_{p_{1}}^{2}\left(\mathcal{E}, p_{2}^{*}(E)\right) .
\end{aligned}
$$

By Lemma 1.10, $\widehat{\mathcal{F}}_{\mathcal{E}}^{2}(E)=\operatorname{Ext}_{p_{1}}^{2}\left(\mathcal{E}, p_{2}^{*}(E)\right)=0$. It is easy to see that

$$
\begin{aligned}
& \operatorname{Hom}_{p_{1}}\left(\mathcal{O}_{\Delta}, p_{2}^{*}(E)\right)=0, \\
& \operatorname{Ext}_{p_{1}}^{1}\left(\mathcal{O}_{\Delta}, p_{2}^{*}(E)\right)=0, \\
& \operatorname{Ext}_{p_{1}}^{2}\left(\mathcal{O}_{\Delta}, p_{2}^{*}(E)\right)=E .
\end{aligned}
$$

Since $\chi(E+\epsilon F+A, E)=0<\chi(E+\epsilon F+A, F)$, the $v+\alpha$-twisted semi-stability of $E$ and $F$ imply that $\widehat{\mathcal{F}}_{\mathcal{E}}^{0}(E)=\operatorname{Hom}(F, E) \otimes F=0$. Therefore $\mathrm{WIT}_{1}$ holds with respect to $\widehat{\mathcal{F}}_{\mathcal{E}}$ and $\widehat{\mathcal{F}}_{\mathcal{E}}^{1}(E)$ is $v+\alpha$-twisted semi-stable. Assume that $\widehat{\mathcal{F}}_{\mathcal{E}}^{1}(E)$ is not $v+\alpha^{-}$-twisted semi-stable. Then there is an exact sequence

$$
0 \rightarrow G_{1} \rightarrow \widehat{\mathcal{F}}_{\mathcal{E}}^{1}(E) \rightarrow G_{2} \rightarrow 0
$$

such that $G_{1}$ is a $v+\alpha^{-}$-twisted stable sheaf with $\operatorname{deg}_{E} G_{1}=0, \chi\left(E-\epsilon F+A, G_{1}\right)>0, \chi\left(E+A, G_{1}\right)=0$ and $G_{2}$ is a $v+\alpha$-twisted semi-stable sheaf. Since $\chi\left(E-\epsilon F+A, G_{1}\right) / \operatorname{rk} G_{1}>0>\chi(E-\epsilon F+A, F) / \operatorname{rk} F$, $\operatorname{Ext}^{2}\left(F, G_{1}\right)=\operatorname{Hom}\left(G_{1}, F\right)^{\vee}=0$. Thus $R^{2} p_{2 *}\left(\mathcal{E} \otimes p_{1}^{*}\left(G_{1}\right)\right)=0$. Since $G_{2}$ is a $v+\alpha$-twisted semi-stable sheaf with $\operatorname{deg}_{E} G_{2}=\chi\left(E+A, G_{2}\right)=0$, Lemma 1.11 implies that $\operatorname{Hom}\left(F, G_{2}\right) \otimes F \rightarrow G_{2}$ is injective, and hence $p_{2 *}\left(\mathcal{E} \otimes p_{1}^{*}\left(G_{2}\right)\right)=0$. Therefore $\mathrm{WIT}_{1}$ holds for $G_{1}$ and $G_{2}$ with respect to $\mathcal{F}_{\mathcal{E}}$. Since $\chi\left(E+\epsilon F+A, \mathcal{F}_{\mathcal{E}}^{1}\left(G_{1}\right)\right)>$ 0 , we get a contradiction.

The last claim (3) will easily follow from the above arguments. We omit the proof.

The following is proved in $\mathrm{Y} 1$.

Proposition 1.13. Keep notation as above. Assume that $v+\alpha^{-}$does not lie on walls. Then $\mathcal{F}_{\mathcal{E}}$ induces an isometry $R_{v(F)}: v^{\perp} \rightarrow v^{\perp}$ and the following diagram is commutative.

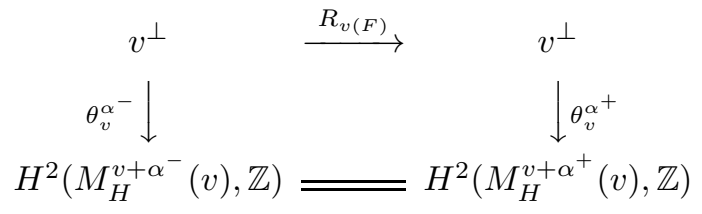

Remark 1.2. If $\alpha$ belongs to exactly one wall $W_{u}, u \in \mathcal{U}$, then there is a $v+\alpha$-twisted stable sheaf $F$ with $v(F)=u$. So we can apply Propositions 1.12 and 1.13 to this $F$. 


\section{Resolution of the Singularities of $\bar{M}_{H}^{v}(v)$}

2.1. Exceptional locus of the resolution. Assume that there is a point $x$ of $\bar{M}_{H}^{v}(v)$ representing a properly $v$-twisted semi-stable sheaf. Assume that $x$ is represented by an $S$-equivalence class $\bigoplus_{i=0}^{n} E_{i}^{\oplus a_{i}}$, where $E_{i}$ is a $v$-twisted stable sheaf such that $\left\langle v\left(E_{i}\right), \widehat{H}\right\rangle=\left\langle v\left(E_{i}\right), v\right\rangle=0$ and $E_{i} \neq E_{j}$ for $i \neq j$. We set $v_{i}:=v\left(E_{i}\right)$.

Lemma 2.1. $\mathbb{Z} v_{0}+\mathbb{Z} v_{1}+\cdots+\mathbb{Z} v_{n}$ is a negative semi-definite lattice of affine type $\tilde{A}_{n}, \tilde{D}_{n}, \tilde{E}_{n}$. More precisely, $\left(-\left\langle v_{i}, v_{j}\right\rangle\right)_{i, j=0}^{n}$ is the Cartan matrix of the affine Lie algebra $\tilde{A}_{n}, \tilde{D}_{n}, \tilde{E}_{n}$. In particular, $v_{0}, v_{1}, \ldots, v_{n}$ are linearly independent.

Proof. We note that

$$
\left\{\begin{array}{l}
\left\langle v_{i}^{2}\right\rangle=-2 \\
\left\langle v_{i}, v_{j}\right\rangle \geq 0, i \neq j \\
\left\langle v, v_{i}\right\rangle=\left\langle\widehat{H}, v_{i}\right\rangle=0 .
\end{array}\right.
$$

If there is a decomposition $\{0,1, \ldots, n\}=I \amalg J$ such that $\left\langle v_{i}, v_{j}\right\rangle=0$ for all $i \in I, j \in J$, then $0=$ $\left\langle v^{2}\right\rangle=\left\langle\left(\sum_{i \in I} a_{i} v_{i}\right)^{2}\right\rangle+\left\langle\left(\sum_{j \in J} a_{j} v_{j}\right)^{2}\right\rangle$. By (1.11), $\sum_{i \in I} a_{i} v_{i}=\sum_{j \in J} a_{j} v_{j}=0$ in $v^{\perp} / \mathbb{Z} v$. Since $0 \leq$ $\operatorname{rk}\left(\sum_{i \in I} a_{i} v_{i}\right), \operatorname{rk}\left(\sum_{j \in J} a_{j} v_{j}\right) \leq \operatorname{rk} v$, we get $I=\emptyset$ or $J=\emptyset$. Then as in the classification of the singular fiber of an elliptic surface, we get our claim.

Assume that there is another point $x^{\prime}$ of $\bar{M}_{H}^{v}(v)$ representing a properly $v$-twisted semi-stable sheaf $\bigoplus_{i=0}^{n^{\prime}} E_{i}^{\prime \oplus a_{i}^{\prime}}$, where $E_{i}^{\prime}$ is a $v$-twisted stable sheaf such that $\left\langle v\left(E_{i}^{\prime}\right), \widehat{H}\right\rangle=\left\langle v\left(E_{i}^{\prime}\right), v\right\rangle=0$ and $E_{i}^{\prime} \neq E_{j}^{\prime}$ for $i \neq j$. We set $v_{i}^{\prime}:=v\left(E_{i}^{\prime}\right)$. Then we have the following lemma.

\section{Lemma 2.2.}

$$
\left(\mathbb{Z} v_{0}+\mathbb{Z} v_{1}+\cdots+\mathbb{Z} v_{n}\right) \perp\left(\mathbb{Z} v_{0}^{\prime}+\mathbb{Z} v_{1}^{\prime}+\cdots+\mathbb{Z} v_{n^{\prime}}^{\prime}\right)
$$

Proof. We set

$$
\begin{aligned}
& S_{1}:=\left\{i \mid v_{i}^{\prime} \in\left(\mathbb{Z} v_{0}+\mathbb{Z} v_{1}+\cdots+\mathbb{Z} v_{n}\right)^{\perp}\right\}, \\
& S_{2}:=\left\{0,1, \ldots, n^{\prime}\right\} \backslash S_{1} .
\end{aligned}
$$

Then $v=\sum_{i \in S_{1}} a_{i}^{\prime} v_{i}^{\prime}+\sum_{i \in S_{2}} a_{i}^{\prime} v_{i}^{\prime}$. Assume that $i \in S_{2}$. Since $0=\left\langle v_{i}^{\prime}, v\right\rangle=\sum_{j} a_{j}\left\langle v_{i}^{\prime}, v_{j}\right\rangle,\left\langle v_{i}^{\prime}, v_{j}\right\rangle<0$ for some $j$. Then $\chi\left(E_{i}^{\prime}, E_{j}\right)>0$, which implies that there is a non-zero homomorphism $E_{i}^{\prime} \rightarrow E_{j}$ or $E_{j} \rightarrow$ $E_{i}^{\prime}$. Since $E_{i}^{\prime}$ and $E_{j}$ are $v$-twisted stable sheaves such that $\chi\left(E, E_{i}^{\prime}(n H)\right) / \operatorname{rk} E_{i}^{\prime}=\chi\left(E, E_{j}(n H)\right) / \operatorname{rk} E_{j}(=$ $\chi(E, E(n H)) / \mathrm{rk} E)$ for all $n$, we get $E_{i}^{\prime} \cong E_{j}$. Thus $v_{i}^{\prime} \in\left\{v_{0}, v_{1}, \ldots, v_{n}\right\}$. Then we get that $\left\langle\left(\sum_{i \in S_{1}} a_{i}^{\prime} v_{i}^{\prime}\right)^{2}\right\rangle+$ $\left\langle\left(\sum_{i \in S_{2}} a_{i}^{\prime} v_{i}^{\prime}\right)^{2}\right\rangle=0$, and hence $\sum_{i \in S_{1}} a_{i}^{\prime} v_{i}^{\prime}, \sum_{i \in S_{2}} a_{i}^{\prime} v_{i}^{\prime} \in \mathbb{Z} v$. Since $\operatorname{rk}\left(\sum_{i \in S_{2}} a_{i}^{\prime} v_{i}^{\prime}\right) \leq \operatorname{rk} v, \sum_{i \in S_{2}} a_{i}^{\prime} v_{i}^{\prime}=0$ or $v$. If $\sum_{i \in S_{2}} a_{i}^{\prime} v_{i}^{\prime}=v$, then Lemma 2.1 implies that $S_{2}=\{0,1, \ldots, n\}$ and $a_{i}^{\prime}=a_{i}$, which implies that $x^{\prime}=x$. Since $x \neq x^{\prime}$, we get that $S_{1}=\left\{0,1, \ldots, n^{\prime}\right\}$. Thus our claim holds.

We shall study the fiber of $\phi_{\alpha}: M_{H}^{v+\alpha}(v) \rightarrow \bar{M}_{H}^{v}(v)$ at $x$. By the classification of the (extended) Dynkin diagram, we may assume that $a_{0}=1$. Then $v_{i}, i=1,2, \ldots, n$ become a fundamental root system of the corresponding finite Lie algebra $\mathfrak{g}$ under the change of the sign of the bilinear form.

\section{Lemma 2.3.}

$$
\mathcal{U}^{\prime}=\left(\mathcal{U}^{\prime} \cap\left(\bigoplus_{i=0}^{n} \mathbb{Z} v_{i}\right)^{\perp}\right) \coprod\left(\mathcal{U}^{\prime} \cap \bigoplus_{i=0}^{n} \mathbb{Z} v_{i}\right)
$$

and

$$
\begin{aligned}
\mathcal{U}^{\prime} \cap\left(\bigoplus_{i=0}^{n} \mathbb{Z} v_{i}\right) & =\left\{u \in \bigoplus_{i=0}^{n} \mathbb{Z} v_{i} \mid\left\langle u^{2}\right\rangle=-2,0<\operatorname{rk} u<\operatorname{rk} v\right\} \\
& =\Psi_{+} \coprod\left(v-\Psi_{+}\right)
\end{aligned}
$$

where $\Psi_{+}:=\left\{u=\sum_{i=1}^{n} b_{i} v_{i} \mid\left\langle u^{2}\right\rangle=-2, b_{i} \geq 0\right\}$ is the set of positive roots of $\mathfrak{g}$.

Proof. For $u \in \mathcal{U}^{\prime}$, we set $w=v-u$. Since $\langle v, u\rangle=0$, we get $\left\langle w^{2}\right\rangle=\left\langle u^{2}\right\rangle=-2$. Since $0<\operatorname{rk} u<\operatorname{rk} v$, we have $\mathrm{rk} w>0$. By Proposition 1.2, there are $v$-twisted semi-stable sheaves $F$ and $G$ with $v(F)=u$ and $v(G)=w$. Applying Lemma 2.2 to $F \oplus G$, we see that $u=\sum_{i=0}^{n} b_{i} v_{i} \in \bigoplus_{i=0}^{n} \mathbb{Z} v_{i}, a_{i} \geq b_{i} \geq 0$ or $u \in\left(\bigoplus_{i=0}^{n} \mathbb{Z} v_{i}\right)^{\perp}$ according as $F \oplus G$ is $S$-equivalent to $\bigoplus_{i=0}^{n} E_{i}^{\oplus a_{i}}$ or not. Thus the first claim holds. If $b_{0}=0$, then $u \in \Psi_{+}$and if $b_{0}=1$, then $w \in \Psi_{+}$. Thus $u=v-w \in v-\Psi_{+}$. Therefore the second assertion also holds. 
Therefore the wall $W_{u}$ corresponds to the wall defining the Weyl chamber. More precisely, $W_{u} \cap$ $\left(\left(\bigoplus_{i=0}^{n} \mathbb{Z} v_{i}\right) / \mathbb{Z} v\right) \otimes \mathbb{R}$ is the corresponding wall. We define the fundamental Weyl chamber:

$$
D:=\left\{\alpha \in \delta\left(H^{\perp}\right)_{a l g} \otimes_{\mathbb{Q}} \mathbb{R} \mid\left\langle v_{i}, \alpha\right\rangle>0, i>0\right\} .
$$

For a small $\alpha \in D$, we describe the exceptional set $\phi_{\alpha}^{-1}(x)$. The method is the same as in Y1.

Lemma 2.4. Assume that $\alpha \in \delta\left(H^{\perp}\right)_{\text {alg }}$ belongs to $D$ and $\left|\left\langle\alpha^{2}\right\rangle\right| \ll 1$. Let $F$ be a $v+\alpha$-twisted semi-stable sheaf such that $v(F)=v_{0}+\sum_{j>0} b_{j} v_{j}, 0 \leq b_{j} \leq a_{j}$.

(1) If $v(F) \neq v$, then $F$ is $v+\alpha$-twisted stable and $F$ is $S$-equivalent to $E_{0} \oplus\left(\bigoplus_{j>0} E_{j}^{\oplus b_{j}}\right)$ with respect to the $v$-twisted stability.

(2) For a non-zero homomorphsim $\phi: E_{i} \rightarrow F, i>0, \phi$ is injective and $F^{\prime}:=\operatorname{coker} \phi$ is a $v+\alpha$-twisted stable sheaf.

(3) If there is a non-trivial extension

$$
0 \rightarrow E_{i} \rightarrow F^{\prime \prime} \rightarrow F \rightarrow 0
$$

and $b_{i}+1 \leq a_{i}$, then $F^{\prime \prime}$ is $v+\alpha$-twisted stable.

Proof. We take elements $E, A \in K(X) \otimes \mathbb{Q}$ such that $v(E)=v, v(A)=\alpha$. Since $\left|\left\langle\alpha^{2}\right\rangle\right| \ll 1, F$ is $v$ twisted semi-stable. Assume that $F$ is $S$-equivalent to $\bigoplus_{j=0}^{n^{\prime}} E_{j}^{\prime \oplus b_{j}^{\prime}}$ with respect to the $v$-twisted stability. Since $F \oplus\left(\bigoplus_{j>0} E_{j}^{\oplus\left(a_{j}-b_{j}\right)}\right)$ is $S$-equivalent to $\bigoplus_{j=0}^{n^{\prime}}{E_{j}^{\prime}}^{\oplus b_{j}^{\prime}} \oplus\left(\bigoplus_{j>0} E_{j}^{\oplus\left(a_{j}-b_{j}\right)}\right)$, by Lemma 2.1 and Lemma 2.2. we get that $F$ is $S$-equivalent to $E_{0} \oplus\left(\bigoplus_{j>0} E_{j}^{\oplus b_{j}}\right)$ with respect to the $v$-twisted stability. Since $\chi(E+A, F) \geq \chi(E+A, E)=0$ and $\chi\left(E+A, E_{i}\right)<0$ for all $i>0$, there is no proper subsheaf $E^{\prime}$ such that $\chi\left(E+A, E^{\prime}(n H)\right) / \mathrm{rk} E^{\prime}=\chi(E+A, F(n H)) / \mathrm{rk} F$ for all $n$. Thus the claim (1) holds.

We next prove (2). Since $E_{i}$ is $v$-twisted stable and $F$ is $v$-twisted semi-stable, $\phi$ is injective and $F^{\prime}$ is a $v$-twisted semi-stable sheaf. By $(1), F^{\prime}$ is $S$-equivalent to $E_{0} \oplus\left(\bigoplus_{j>0} E_{j}^{\oplus c_{j}}\right)$ with respect to the $v$ twisted stability, where $v\left(F^{\prime}\right)=v_{0}+\sum_{j>0} c_{j} v_{j}$. If $F^{\prime}$ is not $v+\alpha$-twisted stable, then there is a quotient sheaf $F^{\prime} \rightarrow G$ such that $\operatorname{deg}_{E} G=0$ and $\chi(E+A, G) / \operatorname{rk} G<\chi\left(E+A, F^{\prime}\right) / \operatorname{rk} F^{\prime}$. Since $\left|\left\langle\alpha^{2}\right\rangle\right| \ll 1$ and $\chi\left(E+A, E_{i}\right)<0$ for all $i>0$, we see that $G$ is $v$-twisted semi-stable and is $S$-equivalent to $\bigoplus_{j>0} E_{j}^{\oplus c_{j}^{\prime}}$ with respect to the $v$-twisted stability. Hence we get that $\chi(E+A, G)<0$, which implies that $F$ is not $v+\alpha$-twisted stable. Therefore $F^{\prime}$ is $v+\alpha$-twisted stable.

Finally we prove (3). By our assumption, $\chi\left(E+A, F^{\prime \prime}\right) \geq \chi(E+A, E)=0$. If $F^{\prime \prime}$ is not $v+\alpha$-twisted stable, then there is a quotient sheaf $F^{\prime \prime} \rightarrow G$ such that $\operatorname{deg}_{E} G=0$ and $\chi(E+A, G) / \operatorname{rk} G<\chi\left(E+A, F^{\prime \prime}\right) / \operatorname{rk} F^{\prime \prime}$. Then we see that $G$ is $v$-twisted semi-stable and is $S$-equivalent to $\bigoplus_{j>0} E_{j}^{\oplus c_{j}^{\prime \prime}}$ with respect to the $v$-twisted stability. Then there is a quotient $G \rightarrow E_{j}, j>0$. By (2.15), we have an exact sequence

$$
0=\operatorname{Hom}\left(F, E_{j}\right) \rightarrow \operatorname{Hom}\left(F^{\prime \prime}, E_{j}\right) \rightarrow \operatorname{Hom}\left(E_{i}, E_{j}\right) .
$$

We consider the map $\psi: E_{i} \rightarrow F^{\prime \prime} \rightarrow G \rightarrow E_{j}$. Then $\psi$ is an isomorphism, which implies that the extension (2.7) splits.

By Lemma 2.4 (1), we get the following corollary.

Corollary 2.5. We set $w:=v_{0}+\sum_{j>0} b_{j} v_{j}, 0 \leq b_{j} \leq a_{j}$. If $w \neq v$, then $\bar{M}_{H}^{v+\alpha}(w)=M_{H}^{v+\alpha}(w)$. In particular, if $\left\langle w^{2}\right\rangle=-2$, then $M_{H}^{v+\alpha}(w)$ is not empty and consists of one element.

Corollary 2.6. Let $F$ be a $v+\alpha$-twisted stable sheaf with $v(F)=v_{0}+\sum_{j>0} b_{j} v_{j}, 0 \leq b_{j} \leq a_{j}$.

(1) If $v(F)=v$, then $\operatorname{dim} \operatorname{Hom}\left(E_{i}, F\right) \leq 1$.

(2) If $v(F) \neq v$, then $\operatorname{dim} \operatorname{Hom}\left(E_{i}, F\right)=\max \left\{-\left\langle v(F), v_{i}\right\rangle, 0\right\}$.

Proof. We set $\operatorname{dim} \operatorname{Hom}\left(E_{i}, F\right)=k$. By the Riemann-Roch theorem, $k \geq-\left\langle v(F), v_{i}\right\rangle$. Hence if $k=0$, then our claims (1), (2) hold. Assume that $k>0$. By Lemma 2.4, $\phi: \operatorname{Hom}\left(E_{i}, F\right) \otimes E_{i} \rightarrow F$ is injective and $F^{\prime}:=$ coker $\phi$ is $v+\alpha$-twisted stable. If $v(F)=v$, then $-2 \leq\left\langle v\left(F^{\prime}\right)^{2}\right\rangle=-2 k^{2}$. Hence $k \leq 1$. If $v(F) \neq v$, then $\left\langle v(F)^{2}\right\rangle=-2$ and hence $-2 \leq\left\langle v\left(F^{\prime}\right)^{2}\right\rangle=-2-2 k\left(k+\left\langle v(F), v_{i}\right\rangle\right)$. Then $k+\left\langle v(F), v_{i}\right\rangle \leq 0$, which implies that $k=-\left\langle v(F), v_{i}\right\rangle$.

Corollary 2.7. We set $w=v_{0}+\sum_{j>0} b_{j} v_{j}, 0 \leq b_{j} \leq a_{j}$. If $\left\langle w^{2}\right\rangle=\left\langle\left(w-v_{i}\right)^{2}\right\rangle=-2$, then we have an isomorphism $M_{H}^{v+\alpha}(w) \rightarrow M_{H}^{v+\alpha}\left(w-v_{i}\right)$ sending $F$ to $\operatorname{coker}\left(E_{i} \rightarrow F\right)$.

Proof. By our assumption, we see that $\left\langle w, v_{i}\right\rangle=-1$. By Corollary 2.6. $\operatorname{Hom}\left(E_{i}, F\right)=\mathbb{C}$. By Lemma 2.4 $(2), F^{\prime}:=\operatorname{coker}\left(E_{i} \rightarrow F\right)$ is a $v+\alpha$-twisted stable sheaf with $v\left(F^{\prime}\right)=w-v_{i}$. Conversely for a $v+\alpha$-twisted stable sheaf $F^{\prime}$ with $v\left(F^{\prime}\right)=w-v_{i}$, we get $\left\langle w-v_{i}, v_{i}\right\rangle=1$, and hence by Corollary 2.6 and Lemma 2.4 (3), the non-trivial extension of $F^{\prime}$ by $E_{i}$ gives a $v+\alpha$-twisted stable sheaf $F$ with $v(F)=w$. 
We set

$$
\begin{aligned}
C_{i} & :=\left\{(E, U) \mid \begin{array}{l}
E \in M_{H}^{v+\alpha}(v), U \subset \operatorname{Hom}\left(E_{i}, E\right) \\
\operatorname{dim} U=1
\end{array}\right\} \\
& =\left\{E_{i} \subset E \mid E \in M_{H}^{v+\alpha}(v)\right\} .
\end{aligned}
$$

$C_{i}$ is the moduli space of twisted coherent systems.

Proposition 2.8. $\quad$ (1) $C_{i} \subset \phi_{\alpha}^{-1}(x)$.

(2) $C_{i} \cong \mathbb{P}^{1}$ and the natural map $\pi: C_{i} \rightarrow M_{H}^{v+\alpha}(v)$ is a closed immersion. In particular, $C_{i}$ is not empty.

Proof. We set $F:=\operatorname{coker}\left(E_{i} \rightarrow E\right)$. Then $E$ is $S$-equivalent to $E_{i} \oplus F$ with respect to the $v$-twisted stability. By Lemma 2.4 (1), $F$ is $S$-equivalent to $E_{i}^{\oplus\left(a_{i}-1\right)} \oplus \bigoplus_{j \neq i} E_{j}^{\oplus a_{j}}$, and hence the first claim holds. We next show the assertion (2). We note that the Zariski tangent space of $C_{i}$ at $E_{i} \rightarrow E$ is

$$
\operatorname{Ext}^{1}\left(E_{i} \rightarrow E, E\right)=\operatorname{Ext}^{1}(F, E)
$$

and the obstruction for the infinitesimal lifting belongs to

$$
\operatorname{ker}\left(\operatorname{Ext}^{2}\left(E_{i} \rightarrow E, E\right) \rightarrow \operatorname{Ext}^{2}(E, E) \stackrel{t r}{\rightarrow} H^{2}\left(X, \mathcal{O}_{X}\right)\right) .
$$

We shall first show that $C_{i}$ is smooth at $E_{i} \subset E$. Since $\operatorname{Ext}^{2}\left(E_{i} \rightarrow E, E\right)=\operatorname{Ext}^{2}(F, E)=\operatorname{Hom}(E, F)^{\vee}$, it is sufficient to show that $\operatorname{Hom}(E, F)=\mathbb{C}$. By the exact sequence

$$
0 \rightarrow E_{i} \rightarrow E \rightarrow F \rightarrow 0
$$

we get an exact sequence

$$
0 \rightarrow \operatorname{Hom}(F, F) \rightarrow \operatorname{Hom}(E, F) \rightarrow \operatorname{Hom}\left(E_{i}, F\right) .
$$

If $\operatorname{Hom}\left(E_{i}, F\right) \neq 0$, then we get $\operatorname{dim} \operatorname{Hom}\left(E_{i}, E\right) \geq 2$, which contradicts Corollary 2.6. Hence $\operatorname{Hom}(F, F) \cong$ $\operatorname{Hom}(E, F)$. By Lemma $2.4(2), F$ is simple. Therefore $\operatorname{Hom}(E, F)=\mathbb{C}$. Since the homomorphism

$$
\operatorname{Ext}^{1}\left(E_{i} \rightarrow E, E\right) \rightarrow \operatorname{Ext}^{1}(E, E)
$$

between the Zariski tangent spaces is injective, $C_{i} \rightarrow M_{H}^{v+\alpha}(v)$ is a closed immersion, provided that $C_{i} \neq \emptyset$.

We next show that $C_{i} \neq \emptyset$ and isomorphic to $\mathbb{P}^{1}$. Since $\left\langle\left(v-v_{i}\right)^{2}\right\rangle=-2$, Corollary 2.5 implies that $M_{H}^{v+\alpha}(v)$ consists of exactly one $v+\alpha$-twisted stable sheaf $F$. By Corollary $2.6(2), \operatorname{Hom}\left(E_{i}, F\right)=0$. Thus $\operatorname{Ext}^{2}\left(F, E_{i}\right)=\operatorname{Hom}\left(E_{i}, F\right)^{\vee}=0$. Since $\operatorname{Hom}\left(F, E_{i}\right)=0$, we get $\operatorname{Ext}^{1}\left(F, E_{i}\right) \cong \mathbb{C}^{\oplus 2}$. Let $E$ be a coherent sheaf which is defined by a non-trivial extension

$$
0 \rightarrow E_{i} \rightarrow E \rightarrow F \rightarrow 0 .
$$

By Lemma 2.4 (3), $E$ is $v+\alpha$-twisted stable. Therefore $C_{i} \neq \emptyset$ and $C_{i} \cong \mathbb{P}^{1}$.

Proposition 2.9. We identify $C_{i}$ with its image $\pi\left(C_{i}\right)$. Then we have

$$
\phi_{\alpha}^{-1}(x)=\cup_{i=1}^{n} C_{i}
$$

and $\cup_{i=1}^{n} C_{i}$ is a simple normal crossing divisor.

(2) $\left(C_{i}, C_{j}\right)=\left\langle v_{i}, v_{j}\right\rangle$. In particular the dual graph of $C_{i}, 1 \leq i \leq n$ is of type $A_{n}, D_{n}, E_{n}$.

Proof. By Proposition 2.8 (1), $\phi_{\alpha}^{-1}(x) \supset \cup_{i=1}^{n} C_{i}$. Since $\alpha \in D$, we get $\phi_{\alpha}^{-1}(x)=\cup_{i=1}^{n} C_{i}$. We shall study the configuration of $C_{i}, 1 \leq i \leq n$. Here we give a geometric argument based on Lemma 2.4. We shall prove the following assertions:

(i) $C_{i} \cap C_{j} \neq \emptyset$ if and only if $\left\langle v_{i}, v_{j}\right\rangle=1$.

(ii) If $C_{i} \cap C_{j} \neq \emptyset$, then $\#\left(C_{i} \cap C_{j}\right)=1$.

(iii) If $C_{i} \cap C_{j} \neq \emptyset$, then $C_{i}$ and $C_{j}$ intersect transversely.

(iv) $C_{i} \cap C_{j} \cap C_{k}=\emptyset$ for three curves $C_{i}, C_{j}, C_{k}$.

(i) Assume that $C_{i} \cap C_{j} \neq \emptyset$ and take a point $E \in C_{i} \cap C_{j}$. Then $E$ fits in an exact sequence

$$
0 \rightarrow E_{i} \oplus E_{j} \rightarrow E \rightarrow F^{\prime} \rightarrow 0
$$

By Lemma 2.4 (2), $F^{\prime}$ is a $v+\alpha$-twisted stable sheaf. Since $\delta\left(H^{\perp}\right)_{a l g}$ is negative definite, $-2 \leq\left\langle v\left(F^{\prime}\right)^{2}\right\rangle<0$. Thus $\left\langle v\left(F^{\prime}\right)^{2}\right\rangle=-2$. Then $-2=\left\langle v\left(F^{\prime}\right)^{2}\right\rangle=-4+2\left\langle v\left(E_{i}\right), v\left(E_{j}\right)\right\rangle$, which implies that $\left\langle v\left(E_{i}\right), v\left(E_{j}\right)\right\rangle=1$. Conversely if $\left\langle v\left(E_{i}\right), v\left(E_{j}\right)\right\rangle=1$, then $\left\langle\left(v-\left(v_{i}+v_{j}\right)\right)^{2}\right\rangle=-2$. Hence there is a $v+\alpha$-twisted semi-stable sheaf $F^{\prime}$ with $v\left(F^{\prime}\right)=v-\left(v_{i}+v_{j}\right)$. By Lemma $2.4(1), F^{\prime}$ is $v+\alpha$-twisted stable. By Corollary 2.6 (2), $\operatorname{Hom}\left(E_{i}, F^{\prime}\right)=0$. Hence $\operatorname{dim} \operatorname{Ext}^{1}\left(E_{i}, F^{\prime}\right)=\left\langle v_{i}, v-\left(v_{i}+v_{j}\right)\right\rangle=1$. We also have $\operatorname{dim} \operatorname{Ext}^{1}\left(E_{j}, F^{\prime}\right)=1$. We take an extension

$$
0 \rightarrow E_{i} \oplus E_{j} \rightarrow E \rightarrow F^{\prime} \rightarrow 0
$$


whose extension class is given by $\left(e_{i}, e_{j}\right) \in \operatorname{Ext}^{1}\left(E_{i}, F^{\prime}\right) \oplus \operatorname{Ext}^{1}\left(E_{j}, F^{\prime}\right), e_{i}, e_{j} \neq 0$. Then Lemma 2.4 (3) implies that $E$ is a $v+\alpha$-twisted stable sheaf with $v(E)=v$. Therefore $C_{i}$ and $C_{j}$ intersect at $E$.

(ii) Assume that $C_{i} \cap C_{j} \neq \emptyset$. Then every member of $C_{i} \cap C_{j}$ fits in an extension (2.18). Since $M_{H}^{v+\alpha}(v-$ $\left.\left(v_{i}+v_{j}\right)\right)=\left\{F^{\prime}\right\}$ and $E$ does not depend on the choice of $\left(e_{i}, e_{j}\right)$, we get $\#\left(C_{i} \cap C_{j}\right)=1$.

(iii) Assume that $C_{i}$ and $C_{j}$ intersect at $E$. Since $\operatorname{Hom}\left(E_{i} \oplus E_{j}, F^{\prime}\right)=0$ and $F^{\prime}$ is $v+\alpha$-twisted stable, $\operatorname{Ext}^{2}\left(F^{\prime}, E\right)=\operatorname{Hom}\left(E, F^{\prime}\right)^{\vee}=\mathbb{C}$. Then we see that the natural homomorphism

$$
\operatorname{Ext}^{1}\left(E / E_{i}, E\right) \oplus \operatorname{Ext}^{1}\left(E / E_{j}, E\right) \rightarrow \operatorname{Ext}^{1}(E, E)
$$

of tangent spaces is an isomorphism, and hence $C_{i}$ and $C_{j}$ intersect transversely.

(iv) If $C_{i} \cap C_{j} \cap C_{k} \neq \emptyset$, then $\left\langle v_{i}, v_{j}\right\rangle=\left\langle v_{j}, v_{k}\right\rangle=\left\langle v_{k}, v_{i}\right\rangle=1$, which implies that $\left\langle\left(v_{i}+v_{j}+v_{k}\right)^{2}\right\rangle=0$. Since $\delta\left(H^{\perp}\right)_{\text {alg }}$ is negative definite, this is impossible.

Therefore $\cup_{i} C_{i}$ is simple normal crossing and $\left(C_{i}, C_{j}\right)=\left\langle v_{i}, v_{j}\right\rangle$.

Lemma 2.10. Let $\left[C_{i}\right] \in H_{2}\left(M_{H}^{v+\alpha}(v), \mathbb{Z}\right)$ be the fundamental class of $C_{i}$ and $\operatorname{PD}\left(\left[C_{i}\right]\right) \in H^{2}\left(M_{H}^{v+\alpha}(v), \mathbb{Z}\right)$ the Poincaré dual of $\left[C_{i}\right]$. Then

$$
\mathrm{PD}\left(\left[C_{i}\right]\right)=\theta_{v}^{\alpha}\left(-v_{i}\right)
$$

Proof. Let $M_{H}^{v+\alpha}(v)=\cup_{\lambda} U_{\lambda}$ be an analytic open covering and $\mathcal{F}_{\lambda}$ a local universal family on $U_{\lambda} \times X$. Then $\operatorname{Hom}_{p_{U_{\lambda}}}\left(p_{X}^{*}\left(E_{i}\right), \mathcal{F}_{\lambda}\right)=\operatorname{Ext}_{p_{U_{\lambda}}}^{2}\left(p_{X}^{*}\left(E_{i}\right), \mathcal{F}_{\lambda}\right)=0$ and $C_{i} \cap U_{\lambda}$ is the scheme-theoretic support of $\operatorname{Ext}_{p_{U_{\lambda}}}^{1}\left(p_{X}^{*}\left(E_{i}\right), \mathcal{F}_{\lambda}\right)$, where $p_{X}: U_{\lambda} \times X \rightarrow X$ and $p_{U_{\lambda}}: U_{\lambda} \times X \rightarrow U_{\lambda}$ are projections. For a sufficiently large integer $n, V_{\lambda}:=p_{U_{\lambda} *}\left(\mathcal{F}_{\lambda} \otimes p_{X}^{*}\left(\mathcal{O}_{X}(n H)\right)\right)$ is a locally free sheaf on $U_{\lambda}$. Then we can glue $\left\{\mathcal{F}_{\lambda} \otimes p_{U_{\lambda}}^{*}\left(V_{\lambda}^{\vee}\right)\right\}$ together and we get a quasi-universal family $\mathcal{F}$ on $M_{H}^{v+\alpha}(v) \times X$. By using the Grothendieck Riemann-Roch theorem, we get that the Poincaré dual of $\left[C_{i}\right]$ is $\theta_{v}^{\alpha}\left(-v_{i}\right)$.

Remark 2.1. By Lemma 2.10, the non-emptyness of $C_{i}$ also follows from the fact that $\theta_{v}^{\alpha}\left(v_{i}\right) \neq 0$. Since $\theta_{v}^{\alpha}$ is an isometry, the configuration of $C_{i}$ is also described by the configuration of $v_{i}$. In particular, we get a different proof of Proposition 2.9.

Since $\phi_{\alpha}^{-1}(x)$ is a union of $(-2)$-curves, we get the following proposition.

Proposition 2.11. $\phi_{\alpha}: M_{H}^{v+\alpha}(v) \rightarrow \bar{M}_{H}^{v}(v)$ is surjective and $\bar{M}_{H}^{v}(v)$ contains a $v$-twisted stable sheaf.

Proof. Let $x$ be a point of $\bar{M}_{H}^{v}(v)$ and assume that $x$ corresponds to a properly $v$-twisted semi-stable sheaf. Then $\phi_{\alpha}^{-1}(x) \neq \emptyset$ with $\operatorname{dim} \phi_{\alpha}^{-1}(x)=1$. Since $\phi_{\alpha}: \phi_{\alpha}^{-1}\left(M_{H}^{v}(v)\right) \rightarrow M_{H}^{v}(v)$ is an isomorphism and $\bar{M}_{H}^{v}(v) \backslash M_{H}^{v}(v)$ is a finite set, we get our claims.

The remaining of this section is an appendix.

Lemma 2.12. Assume that $\alpha \in D,\left|\left\langle\alpha^{2}\right\rangle\right| \ll 1$ satisfies that

$$
\left\langle v_{i}, \alpha\right\rangle / \operatorname{rk} v_{i}>\left\langle v+\sum_{j>0} a_{j} v_{j}, \alpha\right\rangle / \operatorname{rk}\left(v+\sum_{j>0} a_{j} v_{j}\right)
$$

for all $i>0$. Let $F$ be a $v+\alpha$-twisted semi-stable sheaf such that $v(F)=v+\sum_{j>0} b_{j} v_{j}, 0 \leq b_{j} \leq a_{j}$. Then

(1) $F$ is $v+\alpha$-twisted stable.

(2) Let $F^{\prime}$ be a coherent sheaf which fits in a non-trivial extension

$$
0 \rightarrow E_{i} \rightarrow F^{\prime} \rightarrow F \rightarrow 0
$$

and $b_{i}+1 \leq a_{i}$. Then $F^{\prime}$ is $v+\alpha$-twisted stable.

(3) For a subsheaf $E_{i}, i>0$ of $F, F^{\prime}:=F / E_{i}$ is $v+\alpha$-twisted stable.

Proof. We take $E, A \in K(X) \otimes \mathbb{Q}$ with $v(E)=v, v(A)=\alpha$. Let

$$
0=F_{0} \subset F_{1} \subset F_{2} \subset \cdots \subset F_{s}=F
$$

be the Jordan-Hölder filtration of $F$ with respect to the $v$-twisted stability. For $F_{i} / F_{i-1}$ with $F_{i} / F_{i-1} \notin$ $\left\{E_{0}, E_{1}, \ldots, E_{n}\right\}$, a similar argument to the proof of Lemma 2.2 shows that $\operatorname{Ext}^{1}\left(F_{i} / F_{i-1}, E_{j}\right)=0$ for all $j$. Hence replacing the filtration, we may assume that $F_{i} / F_{i-1} \notin\left\{E_{0}, E_{1}, \ldots, E_{n}\right\}$ for $0 \leq i \leq k$ and $F_{i} / F_{i-1} \in$ $\left\{E_{0}, E_{1}, \ldots, E_{n}\right\}$ for $i>k$. Then we get $F=F_{k} \oplus F / F_{k}$ and $-2 \leq\left\langle v(F)^{2}\right\rangle=\left\langle v\left(F_{k}\right)^{2}\right\rangle+\left\langle v\left(F / F_{k}\right)^{2}\right\rangle$. Assume that $F_{k} \neq 0$, that is, $k>0$. Then we see that (i) $v\left(F_{k}\right)=v$ and $\left\langle v\left(F / F_{k}\right)^{2}\right\rangle=-2$, or (ii) $\left\langle v\left(F_{k}\right)^{2}\right\rangle=-2$ and $v\left(F / F_{k}\right) \in \mathbb{Z} v$. If $v\left(F_{k}\right)=v$, then $\left\langle v\left(F_{k}\right), \alpha\right\rangle=0$ and $\left\langle v\left(F / F_{k}\right), \alpha\right\rangle=\sum_{j} b_{j}\left\langle v_{j}, \alpha\right\rangle>0$, which contradicts the $v+\alpha$-twisted semi-stability of $F$. Hence the case (i) does not occur. If the case (ii) occurs, then since $\left\langle v\left(F_{k}\right)^{2}\right\rangle=-2$, we get that $\left\langle v(F), v\left(F_{i} / F_{i-1}\right)\right\rangle=\left\langle v\left(F_{k}\right), v\left(F_{i} / F_{i-1}\right)\right\rangle \neq 0$ for some $i \leq k$. On the other hand, by our choice of $v(F)$, we get $\left\langle v(F), v\left(F_{i} / F_{i-1}\right)\right\rangle=0$ for all $i \leq k$. Hence the case (ii) does not occur. Therefore $F_{k}=0$. Then we see that $F$ is $S$-equivalent to $E_{0} \oplus\left(\bigoplus_{j>0} E_{j}^{\oplus\left(a_{j}+b_{j}\right)}\right)$. 
Our assumption (2.21) implies that

$$
\chi\left(E+A, E_{i}\right) / \mathrm{rk} E_{i}<\chi\left(E+A, 2 E-E_{0}\right) / \operatorname{rk}\left(2 E-E_{0}\right) .
$$

Since $F=2 E-E_{0}-\sum_{j>0} c_{j} E_{j}, 0 \leq c_{j} \leq a_{j}$ as an element of $K(X)$, we get that

$$
\chi\left(E+A, 2 E-E_{0}\right) / \operatorname{rk}\left(2 E-E_{0}\right) \leq \chi(E+A, F) / \mathrm{rk} F .
$$

In the same way as in the proof of Lemma 2.4 (1), we see that $F$ is a $v+\alpha$-twisted stable sheaf. Thus (1) holds. The proof of (2) and (3) are the same as in the proof of Lemma 2.4.

Remark 2.2. If $\left\langle v_{i}, \alpha\right\rangle=\left\langle v_{j}, \alpha\right\rangle$ for all $1 \leq i, j \leq n$, then (2.21) is satisfied.

In the same way as in the proof of Corollary 2.7, we get the following.

Corollary 2.13. Assume that $\alpha \in D,\left|\left\langle\alpha^{2}\right\rangle\right| \ll 1$ satisfies (2.21). We set $w=v+\sum_{j>0} b_{j} v_{j}, 0 \leq b_{j} \leq a_{j}$.

(1) If $w \neq v$, then $\bar{M}_{H}^{v+\alpha}(w)=M_{H}^{v+\alpha}(w)$.

(2) If $\left\langle w^{2}\right\rangle=\left\langle\left(w-v_{i}\right)^{2}\right\rangle=-2$, then we have an isomorphism $M_{H}^{v+\alpha}(w) \rightarrow M_{H}^{v+\alpha}\left(w-v_{i}\right)$ sending $F \in M_{H}^{v+\alpha}(w)$ to $\operatorname{coker}\left(E_{i} \rightarrow F\right)$.

Remark 2.3. Assume that $\alpha \in D,\left|\left\langle\alpha^{2}\right\rangle\right| \ll 1$ satisfies (2.21). We note that $H_{2}\left(\phi_{\alpha}^{-1}(x), \mathbb{C}\right) \rightarrow H_{2}\left(M_{H}^{v+\alpha}(v), \mathbb{C}\right)$ is injective. We can regard $H_{2}\left(\phi_{\alpha}^{-1}(x), \mathbb{C}\right)$ as the Cartan subalgebra of $\mathfrak{g}$. In order to get $\mathfrak{g}$, we set

$$
\Psi:=\left\{u \mid u=\sum_{i=1}^{n} b_{i} v_{i},\left\langle u^{2}\right\rangle=-2\right\}
$$

Let $P\left(w, w-v_{i}\right)$ be the subscheme of $M_{H}^{v+\alpha}(w) \times M_{H}^{v+\alpha}\left(w-v_{i}\right)$ consisting of pairs $(E, F) \in M_{H}^{v+\alpha}(w) \times$ $M_{H}^{v+\alpha}\left(w-v_{i}\right)$ which fits in an exact sequence

$$
0 \rightarrow E_{i} \rightarrow E \rightarrow F \rightarrow 0
$$

Then we can show that $P\left(w, w-v_{i}\right)$ is isomorphic to $\mathbb{P}^{1}$ or a point. As in $[\mathrm{Na}$, we see that there is an action of $\mathfrak{g}$ on $H_{2}\left(M_{H}^{v+\alpha}(v), \mathbb{C}\right) \oplus \bigoplus_{u \in \Psi} H_{0}\left(M_{H}^{v+\alpha}(v+u), \mathbb{C}\right)$ and we have an isomorphism of $\mathfrak{g}$-module:

$$
H_{2}\left(\phi_{\alpha}^{-1}(x), \mathbb{C}\right) \oplus \bigoplus_{u \in \Psi} H_{0}\left(M_{H}^{v+\alpha}(v+u), \mathbb{C}\right) \cong \mathfrak{g} .
$$

For a homology class $[x] \in H_{2+\left\langle w^{2}\right\rangle}\left(M_{H}^{v+\alpha}(w), \mathbb{C}\right)$, the action of Chevalley generators $e_{i}, f_{i}, h_{i}, 1 \leq i \leq n$ are given by

$$
\begin{aligned}
& e_{i}:[x] \mapsto p_{M_{H}^{v+\alpha}\left(w+v_{i}\right) *}\left(\left(M_{H}^{v+\alpha}\left(w+v_{i}\right) \times[x]\right) \cap P\left(w+v_{i}, w\right)\right) \\
& f_{i}:[x] \mapsto(-1)^{t(w)} p_{M_{H}^{v+\alpha}\left(w-v_{i}\right) *}\left(\left([x] \times M_{H}^{v+\alpha}\left(w-v_{i}\right)\right) \cap P\left(w, w-v_{i}\right)\right) \\
& h_{i}:[x] \mapsto-\left\langle w, v_{i}\right\rangle[x],
\end{aligned}
$$

where $p_{M_{H}^{v+\alpha}\left(w+k v_{i}\right)}: M_{H}^{v+\alpha}(w) \times M_{H}^{v+\alpha}\left(w-v_{i}\right) \rightarrow M_{H}^{v+\alpha}\left(w+k v_{i}\right), k=0,-1$ are projections and $t(w)=$ $\left(\operatorname{dim} M_{H}^{v+\alpha}\left(w-v_{i}\right)-\operatorname{dim} M_{H}^{v+\alpha}(w)\right) / 2=-\left(\left\langle w, v_{i}\right\rangle+1\right)$.

\subsection{Other chambers.}

Definition 2.1. Let $W$ be the Weyl group generated by reflections $R_{v_{i}}, i=1,2, \ldots, n$.

$W$ is the Weyl group of $\mathfrak{g}$. By Lemma 2.10 and Proposition 1.13 (also see Remark 1.2), we get the following.

Proposition 2.14. If $\alpha \in w(D), w \in W$, then $\operatorname{PD}\left(\left[C_{i}\right]\right)=\theta_{v}^{\alpha}\left(-w\left(v_{i}\right)\right)$.

We shall give a set-theoretic description of $C_{i}$. Let $M_{H}^{v+\alpha}(v)=\cup_{\lambda} U_{\lambda}$ be an analytic open covering and $\mathcal{F}_{\lambda}$ a local universal family on $U_{\lambda} \times X$. If $\operatorname{rk}\left(w\left(v_{i}\right)\right)=0$, then we set $w\left(v_{i}\right):=\eta+b \rho_{X}$, where $\eta \in \operatorname{Pic}(X)$ satisfies $\left(\eta^{2}\right)=-2$. Then $0=\left\langle\widehat{H}, w\left(v_{i}\right)\right\rangle=(H, \eta)$, which contradicts the ampleness of $H$. Hence $\operatorname{rk} w\left(v_{i}\right) \neq 0$. Let $F_{i}$ be a $v+\alpha$-twisted semi-stable sheaf with $v\left(F_{i}\right)= \pm w\left(v_{i}\right)$. We first assume that $\operatorname{rk}\left(w\left(v_{i}\right)\right)>0$, that is, $v\left(F_{i}\right)=w\left(v_{i}\right)$. Since $\left\langle\alpha, w\left(v_{i}\right)\right\rangle=\left\langle w^{-1}(\alpha), v_{i}\right\rangle>0, \chi\left(E+A, F_{i}\right)=-\left\langle v+\alpha, v\left(F_{i}\right)\right\rangle=-\left\langle\alpha, w\left(v_{i}\right)\right\rangle<0$. Then $\operatorname{Ext}^{2}\left(F_{i}, E\right)=0$ for all $E \in M_{H}^{v+\alpha}(v)$. If $E$ is $v$-twisted stable, then $\operatorname{Hom}\left(F_{i}, E\right)=0$. Hence $L_{\lambda}:=\operatorname{Ext}_{p_{U_{\lambda}}}^{1}\left(p_{X}^{*}\left(F_{i}\right), \mathcal{F}_{\lambda}\right)$ is a torsion sheaf of pure dimension 1 whose support is contained in $\phi_{\alpha}^{-1}(x)$. By the Grothendieck Riemann-Roch theorem, the Poincaré dual of the scheme-theoretic support of $L_{\lambda}$ is $\theta_{v}^{\alpha}\left(-w\left(v_{i}\right)\right)=\operatorname{PD}\left(\left[C_{i}\right]\right)$. Since $H^{0}\left(M_{H}^{v+\alpha}(v), \mathcal{O}_{M_{H}^{v+\alpha}(v)}\left(C_{i}\right)\right)=\mathbb{C}$, we get that

$$
\begin{aligned}
C_{i} & =\left\{E \in M_{H}^{v+\alpha}(v) \mid \operatorname{Ext}^{1}\left(F_{i}, E\right) \neq 0\right\} \\
& =\left\{E \in M_{H}^{v+\alpha}(v) \mid \operatorname{Hom}\left(F_{i}, E\right) \neq 0\right\} .
\end{aligned}
$$


If $\chi\left(E+A, F_{i}\right)>0$, that is, $\operatorname{rk}\left(w\left(v_{i}\right)\right)<0$, then we see that $\operatorname{Hom}\left(F_{i}, E\right)=0$ for all $E \in M_{H}^{v+\alpha}(v)$ and $\operatorname{Ext}^{2}\left(F_{i}, E\right)=0$ for a $v$-twisted stable sheaf $E$. Then we also have $\operatorname{Ext}^{1}\left(F_{i}, E\right)=0$. Hence we see that $\operatorname{Ext}_{p_{U_{\lambda}}}^{1}\left(p_{X}^{*}(F), \mathcal{F}_{\lambda}\right)=0$ and $\operatorname{Ext}_{p_{U_{\lambda}}}^{2}\left(p_{X}^{*}(F), \mathcal{F}_{\lambda}\right)$ is a torsion sheaf of pure dimension 1. Hence we get that

$$
\begin{aligned}
C_{i} & =\left\{E \in M_{H}^{v+\alpha}(v) \mid \operatorname{Ext}^{2}\left(F_{i}, E\right) \neq 0\right\} \\
& =\left\{E \in M_{H}^{v+\alpha}(v) \mid \operatorname{Hom}\left(E, F_{i}\right) \neq 0\right\} .
\end{aligned}
$$

Therefore we get the following proposition.

Proposition 2.15. Assume that $\alpha \in w(D), w \in W$. Then $w\left(v_{i}\right) \neq 0$ for $1 \leq i \leq n$. Let $F_{i}$ be a $v+\alpha$-twisted semi-stable sheaf with $v\left(F_{i}\right)= \pm w\left(v_{i}\right)$ according as the sign of $\operatorname{rk}\left(w\left(v_{i}\right)\right)$. If $\operatorname{rk}\left(w\left(v_{i}\right)\right)>0$, then

$$
C_{i}=\left\{E \in M_{H}^{v+\alpha}(v) \mid \operatorname{Hom}\left(F_{i}, E\right) \neq 0\right\}
$$

If $\operatorname{rk}\left(w\left(v_{i}\right)\right)<0$, then

$$
C_{i}=\left\{E \in M_{H}^{v+\alpha}(v) \mid \operatorname{Hom}\left(E, F_{i}\right) \neq 0\right\}
$$

2.3. Normalness of $\bar{M}_{H}^{v}(v)$.

Proposition 2.16. $\bar{M}_{H}^{v}(v)$ is normal.

Proof. We take $\alpha \in D$ with $\left|\left\langle\alpha^{2}\right\rangle\right| \ll 1$. Let $T$ be a smooth curve and we consider a flat family of polarized $\mathrm{K} 3$ surfaces $\pi:(\mathcal{X}, \mathcal{H}) \rightarrow T$ such that

(i) $\left(\mathcal{X}_{t_{0}}, \mathcal{H}_{t_{0}}\right)=(X, H), t_{0} \in T$,

(ii) there are families of Mukai vectors $\mathbf{v} \in R^{*} \pi_{*} \mathbb{Z}, \mathbf{a} \in R^{*} \pi_{*} \mathbb{Q}$ with $\mathbf{v}_{t_{0}}=v, \mathbf{a}_{t_{0}}=\alpha$ and

(iii) $\operatorname{rk} \operatorname{Pic}\left(\mathcal{X}_{t}\right) \leq 3$ for a point $t \in T$,

where $\left(\mathcal{X}_{t}, \mathcal{H}_{t}\right):=(\mathcal{X} \otimes k(t), \mathcal{H} \otimes k(t))$ and $k(t)$ is the residue field at $t \in T$. Replacing $T$ by a suitable covering of $T$, we may assume that there is a section of $\pi$ and a locally free sheaf $\mathcal{E}$ on $\mathcal{X}$ with $v\left(\mathcal{E}_{t}\right)=\mathbf{v}_{t}$, $t \in T$. We consider the relative quot-scheme $Q:=\operatorname{Quot}_{\mathcal{E}(-n \mathcal{H}) \oplus N / \mathcal{X} / T}^{\mathbf{v}} \rightarrow T$ parametrizing all quotients $\mathcal{E}_{t}\left(-n \mathcal{H}_{t}\right)^{\oplus N} \rightarrow F, t \in T$ with $v(F)=\mathbf{v}_{t}$, where $N:=\chi\left(F\left(n \mathcal{H}_{t}\right)\right)$. We denote the universal quotient sheaf by $\mathcal{F}$. We set

$$
Q^{s s}:=\left\{q \in Q \mid \mathcal{F}_{q}:=\mathcal{F} \otimes k(q) \text { is } \mathbf{v}_{t} \text {-twisted semi-stable with respect to } \mathcal{H}_{t}\right\} .
$$

For $n \gg 0$, we have a relative coarse moduli space $\bar{M}_{\mathcal{X} / T, \mathcal{H}}^{\mathbf{v}}(\mathbf{v}):=Q^{s s} / P G L(N) \rightarrow T$. Since $T$ is defined over a field of characteristic $0, \bar{M}_{\mathcal{X} / T, \mathcal{H}}^{\mathrm{v}}(\mathbf{v})_{t}=\bar{M}_{\mathcal{H}_{t}}^{\mathbf{v}_{t}}\left(\mathbf{v}_{t}\right)$ (cf. MFK, Thm. 1.1]). We also have a relative moduli space $\bar{M}_{\mathcal{X} / T, \mathcal{H}}^{\mathbf{v}+\mathbf{a}}(\mathbf{v}) \rightarrow T$. Replacing $T$ by an open subscheme, we may assume that $\bar{M}_{\mathcal{X} / T, \mathcal{H}}^{\mathbf{v}+\mathbf{v})_{t}}$ consists of $\mathbf{v}_{t}+\mathbf{a}_{t}$-twisted stable sheaves on $\mathcal{X}_{t}$ for all $t \in T$ and there is no walls between $\mathbf{v}_{t}$ and $\mathbf{v}_{t}+\mathbf{a}_{t}$. Then $\bar{M}_{\mathcal{X} / T, \mathcal{H}}^{\mathbf{v}+\mathbf{a}}(\mathbf{v}) \rightarrow T$ is a smooth morphism (Mu1, Thm. 1.17]) and we have a morphism $\Phi: \bar{M}_{\mathcal{X} / T, \mathcal{H}}^{\mathbf{v}+\mathbf{a}}(\mathbf{v}) \rightarrow$ $\bar{M}_{\mathcal{X} / T, \mathcal{H}}^{\mathrm{v}}(\mathbf{v})$.

Claim 2.1. $\bar{M}_{\mathcal{X} / T, \mathcal{H}}^{\mathbf{v}}(\mathbf{v})$ is normal.

Proof of Claim 2.1: It is sufficient to show that $Q^{s s}$ is normal. By Serre's criterion, we shall show that $Q^{s s}$ is Cohen-Macaulay and $Q^{s s}$ is regular in codimension 1 . We first prove that $Q^{s s}$ is Cohen-Macaulay. Let $Q^{s p l}$ be the open subscheme of $Q^{s s}$ parametrizing simple sheaves:

$$
Q^{s p l}:=\left\{q \in Q^{s s} \mid \mathcal{F}_{q} \text { is simple }\right\} .
$$

Then $Q^{s p l} \rightarrow T$ is a smooth morphism ([Mul, Thm. 1.17]). By the usual deformation theory of sheaves and Lemma 2.17 below, $Q_{t}^{s s}$ is a locally complete intersection scheme for all $t$. In particular $Q_{t}^{s s}$ is CohenMacaulay. Since $Q_{t}^{s s}$ is smooth at the generic point, $Q_{t}^{s s}$ is reduced. Let $x_{t}$ be the local parameter of $T$ at $t$. By Lemma 2.18 below, $x_{t}$ is a regular element, which implies that $Q^{s s}$ is flat over $T$. Then $Q^{s s}$ is also Cohen-Macaulay.

We next show that $Q^{s s}$ is regular in codimension 1. It is sufficient to show that $\operatorname{dim}\left(Q^{s s} \backslash Q^{s p l}\right) \leq$ $\operatorname{dim} Q^{s s}-2$. By Lemma 2.17, $\operatorname{dim}\left(Q_{t}^{s s} \backslash Q_{t}^{s p l}\right) \leq \operatorname{dim}\left(Q_{t}^{s s} \backslash Q_{t}^{s}\right) \leq \operatorname{dim} Q_{t}^{s s}-1$ for all $t \in T$. For a point $t \in T$ with $\rho\left(\mathcal{X}_{t}\right) \leq 3$, by a direct computation, we see that $\operatorname{dim}\left(Q_{t}^{s s} \backslash Q_{t}^{s p l}\right) \leq \operatorname{dim} Q_{t}^{s s}-2$. Since $Q^{s p l}$ is an open subscheme of $Q^{s s}$, we get that $\operatorname{dim}\left(Q^{s s} \backslash Q^{s p l}\right) \leq \operatorname{dim} Q^{s s}-2$. Therefore our claim holds.

Since $\Phi$ is a birational morphism, we get

$$
\Phi_{*}\left(\mathcal{O}_{\bar{M}_{\mathcal{X} / T, \mathcal{H}}^{\mathbf{v}}(\mathbf{v})}\right)=\mathcal{O}_{\bar{M}_{\mathcal{X} / T, \mathcal{H}}^{\mathbf{v}}(\mathbf{v})} \cdot
$$

Let $\varphi: \bar{M}_{H}^{v}(v)_{n o r} \rightarrow \bar{M}_{H}^{v}(v)$ be the normalization of $\bar{M}_{H}^{v}(v)$ and $\phi_{\alpha}^{\prime}: M_{H}^{v+\alpha}(v) \rightarrow \bar{M}_{H}^{v}(v)_{n o r}$ the morphism with $\varphi \circ \phi_{\alpha}^{\prime}=\phi_{\alpha}$. Since $\bar{M}_{H}^{v}(v)_{n o r}$ has at worst rational double points as its singularities, we get $R^{1} \phi_{\alpha *}^{\prime} \mathcal{O}_{M_{H}^{v+\alpha}(v)}=0$. Since $\varphi$ is a finite morphism, by the Leray spectral sequence, we get

$$
R^{1} \phi_{\alpha *} \mathcal{O}_{M_{H}^{v+\alpha}(v)}=\varphi_{*}\left(R^{1} \phi_{\alpha *}^{\prime} \mathcal{O}_{M_{H}^{v+\alpha}(v)}\right)=0 .
$$


Since $\bar{M}_{\mathcal{X} / T, \mathcal{H}}^{\mathbf{v}+\mathbf{a}}(\mathbf{v})$ and $\bar{M}_{\mathcal{X} / T, \mathcal{H}}^{\mathbf{v}}(\mathbf{v})$ are flat over $T$, by using the base change theorem, we get

$$
\Phi_{*}\left(\mathcal{O}_{\bar{M}_{\mathcal{X} / T, \mathcal{H}}^{\mathbf{v}}(\mathbf{v})}^{\mathrm{va}}\right) \otimes k\left(t_{0}\right)=\phi_{\mathbf{a}_{t_{0}} *}\left(\mathcal{O}_{\bar{M}_{\mathcal{H}}^{t_{t_{0}}}}^{\mathbf{v}_{t_{0}}+\mathbf{a}_{t_{0}}\left(\mathbf{v}_{t_{0}}\right)}\right) .
$$

Combining this with $(2.36)$, we get $\phi_{\alpha *}\left(\mathcal{O}_{M_{H}^{v+\alpha}(v)}\right)=\mathcal{O}_{\bar{M}_{H}^{v}(v)}$, which implies that $\bar{M}_{H}^{v}(v)$ is normal.

Lemma 2.17. We set

$$
Q_{t}^{s}:=\left\{q \in Q_{t}^{s s} \mid \mathcal{F}_{q} \text { is } \mathbf{v}_{t}+\mathbf{a}_{t} \text {-twisted semi-stable }\right\} .
$$

Then $\operatorname{dim}_{Q_{t}^{s s}}\left(Q_{t}^{s s} \backslash Q_{t}^{s}\right)=\operatorname{dim} Q_{t}^{s}-1$.

Proof. Let $0 \subset F_{1} \subset F_{2} \subset \cdots \subset F_{s}=\mathcal{F}_{q}$ be the Harder-Narasimhan filtration of $\mathcal{F}_{q}$ with respect to the $\mathbf{v}_{t}+\mathbf{a}_{t}$-twisted semi-stability. We set $v_{i}:=v\left(F_{i} / F_{i-1}\right)$. Then $\left\langle\widehat{H}, v_{i}\right\rangle=\left\langle v, v_{i}\right\rangle=0$ and $\left\langle v_{i}^{2}\right\rangle=-2\left(\mathrm{rk} v_{i}\right)^{2}$. We shall compute the dimension of an open subscheme of the flag-scheme $F\left(v_{1}, v_{2}, \ldots, v_{s}\right)$ over $T$ parametrizing filtrations $0 \subset F_{1} \subset F_{2} \subset \cdots \subset F_{s}=\mathcal{F}_{q}, q \in Q_{t}^{s s}$ such that $F_{i} / F_{i-1}, 1 \leq i \leq s$ are $\mathbf{v}_{t}+\mathbf{a}_{t}$-twisted semi-stable sheaves with $v\left(F_{i} / F_{i-1}\right)=v_{i}$. By the arguments in [Y3, sect. 3.3], we get

$$
\operatorname{dim} F\left(v_{1}, v_{2}, \ldots, v_{s}\right)-\operatorname{dim} G L(N)=\sum_{i>j}\left\langle v_{i}, v_{j}\right\rangle+\sum_{i}\left(-\left(\operatorname{rk} v_{i}\right)^{2}\right) .
$$

By the equality

$$
2 \sum_{i>j}\left\langle v_{i}, v_{j}\right\rangle+2 \sum_{i}\left(-\left(\operatorname{rk} v_{i}\right)^{2}\right)=\sum_{i \neq j}\left\langle v_{i}, v_{j}\right\rangle+\sum_{i}\left\langle v_{i}^{2}\right\rangle=\left\langle v^{2}\right\rangle=0,
$$

we get $\operatorname{dim} F\left(v_{1}, v_{2}, \ldots, v_{s}\right)-\operatorname{dim} \operatorname{PGL}(N)=1$. Hence our claim holds.

Lemma 2.18. Let $(A, \mathfrak{m})$ and $(B, \mathfrak{n})$ be Noetherian local rings and $f: A \rightarrow B$ a local homomorphism. Let $x \in \mathfrak{m}$ be a non-zero divisor of $A$ satisfying

(i) $x B=\mathfrak{p}_{1} \cap \mathfrak{p}_{2} \cap \cdots \cap \mathfrak{p}_{n}$ for some prime divisors $\mathfrak{p}_{1}, \mathfrak{p}_{2}, \ldots, \mathfrak{p}_{n}$ of $B$, that is, $B / x B$ is reduced,

(ii) $B_{\mathfrak{p}_{i}}, 1 \leq i \leq n$ are flat over $A$.

Then $x$ is also a non-zero divisor of $B$.

Proof. We set

$$
K:=\left\{a \in B \mid x^{n} a=0 \text { for some positive integer } n\right\}
$$

$K$ is an ideal of $B$ and

$$
\{a \in B \mid x a \in K\}=K \text {. }
$$

We shall prove that $K=0$. By (ii), $K_{\mathfrak{p}_{i}}=0$ for all $i$. Since $(K+x B) / x B$ is a sub $B$-module of $B / x B$ and $B / x B$ is reduced, we get that $(K+x B) / x B=0$. By (2.43), $K=x K$. By Nakayama's lemma, we get $K=0$.

\section{ExAmples}

In this section, we shall give some examples of $\bar{M}_{H}^{v}(v)$ with one singular point. Let $L:=\left(-E_{8}\right)^{\oplus 2} \oplus U^{\oplus 3}$ be the K3 lattice, where $U$ is the hyperbolic lattice.

Lemma 3.1. Let $N$ be an even lattice of signature $(1, s)$ which has a primitive embedding $N \hookrightarrow L$. We set $\Delta(N):=\left\{C \in N \mid\left(C^{2}\right)=-2\right\}$. Assume that there is a primitive element $H$ such that $\left(H^{2}\right)>0$ and $(H, C) \neq 0$ for all $C \in \Delta(N)$. Then there is a K3 surface $X$ and an isometry $f: L \rightarrow H^{2}(X, \mathbb{Z})$ such that $f(N)=\operatorname{Pic}(X)$ and $f(H)$ is ample.

Proof. By the surjectivity of the period map, there is a K3 surface $X$ such that $\operatorname{Pic}(X)=N$. We set $\Delta(X)^{+}:=\{C \in \operatorname{Pic}(X) \mid C$ is a $(-2)$-curve $\}$. By the Picard-Lefschetz reflections, we can find a Hodge isomotry $\phi: H^{2}(X, \mathbb{Z}) \rightarrow H^{2}(X, \mathbb{Z})$ such that $(\phi(f(H)), C)>0$ for all $C \in \Delta(X)^{+}$. Replacing $f$ by $\phi \circ f$, we can choose $f(H)$ to be ample.

Lemma 3.2. Let $\left(a_{i, j}\right)_{i, j=0}^{n}$ be a Cartan matrix of affine type $\tilde{A}_{n}, \tilde{D}_{n}, \tilde{E}_{n}$. Let $N_{1}:=\left(\left(\oplus \oplus_{i=0}^{n} \mathbb{Z} \beta_{i}\right) \oplus \mathbb{Z} \sigma,(\quad, \quad)\right)$ be a lattice such that $\left(\sigma^{2}\right)=0,\left(\sigma, \beta_{0}\right)=1,\left(\sigma, \beta_{i}\right)=0, i>0$ and $\left(\beta_{i}, \beta_{j}\right)=-a_{i, j}$. Assume that there is a primitive embedding $N_{1} \hookrightarrow L$. Then there is a positive integer $d$ and a primitive sublattice $N:=$ $\left(\oplus_{i=0}^{n} \mathbb{Z} \xi_{i},(),\right)$ of $L$ such that $\left(\xi_{i}, \xi_{j}\right)=-a_{i, j}+2 d$.

Proof. Since the signature of $N_{1}$ is $(1, n+1)$, there is a vector $x \in N_{1}^{\perp}$ such that $2 d:=\left(x^{2}\right)>0$. We set $\xi_{i}:=\beta_{i}+x$. Then $\oplus_{i=0}^{n} \mathbb{Z} \xi_{i}$ is a primitive sublattice of $L$ with $\left(\xi_{i}, \xi_{j}\right)=\left(\beta_{i}, \beta_{j}\right)+\left(x^{2}\right)=-a_{i, j}+2 d$.

Remark 3.1. For $\tilde{A}_{n}, \tilde{D}_{n}, n \leq 18$ or $\tilde{E}_{n}$, there is a primitive sublattice $N_{1}$ of $L$ (cf. [S-N]). 
Let $C=\left(a_{i, j}\right)_{i, j=0}^{n}$ be a Cartan matrix of affine type $\tilde{A}_{n}, \tilde{D}_{n}, \tilde{E}_{n}$ and $Q:=\left(\oplus_{i=0}^{n} \mathbb{Z} \alpha_{i},(, \quad)\right)$ the associated root lattice, that is, $\left(\alpha_{i}, \alpha_{j}\right)=a_{i, j}$. Then there is a vector $\delta:=\sum_{i=0}^{n} a_{i} \alpha_{i}, a_{i} \in \mathbb{Z}$ such that

$$
Q^{\perp}:=\{x \in Q \mid(x, y)=0 \text { for all } y \in Q\}=\mathbb{Z} \delta .
$$

By the classification of the Cartan matrix of affine type, we may assume that $a_{0}=1$.

Let $N:=\left(\oplus_{i=0}^{n} \mathbb{Z} \xi_{i},(),\right)$ be a primitive sublattice of $L$ (Lemma 3.2) such that

$$
\left(\xi_{i}, \xi_{j}\right)=-a_{i j}+2 r a,
$$

where $r$ and $a$ are positive integers with $d=r a$. We set

$$
H:=\sum_{i=0}^{n} a_{i} \xi_{i}
$$

Lemma 3.3. (1) $\left(H, \xi_{j}\right)=2 \operatorname{ra}\left(\sum_{i=0}^{n} a_{i}\right)$ for all $j$. In particular, $\left(H^{2}\right)=2 r a\left(\sum_{i=0}^{n} a_{i}\right)^{2}>0$.

(2) $H^{\perp}:=\{\xi \in N \mid(H, \xi)=0\}$ is negative definite and $H^{\perp} \cap \Delta(N)=\emptyset$.

Proof. By (3.1), $\sum_{i=0}^{n} a_{i} a_{i, j}=0$ for all $j$. Then we see that

$$
\left(H, \xi_{j}\right)=\sum_{i=0}^{n} a_{i}\left(\xi_{i}, \xi_{j}\right)=\sum_{i=0}^{n} a_{i}\left(-a_{i j}+2 r a\right)=2 r a\left(\sum_{i=0}^{n} a_{i}\right)
$$

for all $j$. Thus the claim (1) holds. We next show the claim (2). By (3.4), we see that

$$
H^{\perp}=\left\{\sum_{i=0}^{n} d_{i} \xi_{i} \mid d_{i} \in \mathbb{Z}, \sum_{i=0}^{n} d_{i}=0\right\}=\bigoplus_{i=0}^{n-1} \mathbb{Z}\left(\xi_{i}-\xi_{i+1}\right) .
$$

We define a homomorphism

$$
\varphi: H^{\perp} \rightarrow Q=\bigoplus_{i=0}^{n} \mathbb{Z} \alpha_{i}
$$

by sending $\xi_{i}-\xi_{i+1} \in H^{\perp}$ to $\alpha_{i}-\alpha_{i+1} \in Q$. Obviously $\varphi$ is injective and

$$
\operatorname{im} \varphi=\bigoplus_{i=0}^{n-1} \mathbb{Z}\left(\alpha_{i}-\alpha_{i+1}\right)=\left\{\sum_{i=0}^{n} d_{i} \alpha_{i} \mid d_{i} \in \mathbb{Z}, \sum_{i=0}^{n} d_{i}=0\right\} .
$$

By (3.2), we see that

$$
\left(\xi_{i}-\xi_{i+1}, \xi_{j}-\xi_{j+1}\right)=-\left(\alpha_{i}-\alpha_{i+1}, \alpha_{j}-\alpha_{j+1}\right) .
$$

Hence $\varphi$ changes the sign of the bilinear forms. In order to prove our claim, it is sufficient to show the following assertions:

(a) $\operatorname{im} \varphi$ is positive definite.

(b) There is no vector $x \in \operatorname{im} \varphi$ with $\left(x^{2}\right)=2$.

By (3.7), $\delta$ does not belong to $\operatorname{im} \varphi$, which implies that $\operatorname{im} \varphi$ is positive definite. We next prove the claim (b). Since $a_{0}=1$, we can take $\left\{\delta, \alpha_{1}, \alpha_{2}, \ldots, \alpha_{n}\right\}$ as a $\mathbb{Z}$-basis of $Q$. Let $\mathfrak{g}$ be the finite simple Lie algebra whose root lattice is $\oplus_{i=1}^{n} \mathbb{Z} \alpha_{i}$. Assume that an element $x=l \delta+\sum_{i=1}^{n} m_{i} \alpha_{i}, l, m_{i} \in \mathbb{Z}$ satisfies that $\left(x^{2}\right)=2$. Then $\sum_{i=1}^{n} m_{i} \alpha_{i}$ becomes a root of $\mathfrak{g}$. Hence $m_{i} \geq 0$ for all $i$, or $m_{i} \leq 0$ for all $i$. Since $\theta:=\sum_{i=1}^{n} a_{i} \alpha_{i}$ is the highest root of $\mathfrak{g}, \sum_{i=0}^{n} a_{i}>\left|\sum_{i=1}^{n} m_{i}\right|$, and hence we get $l\left(\sum_{i=0}^{n} a_{i}\right)+\sum_{i=1}^{n} m_{i} \neq 0$. Thus $x$ does not belong to $\operatorname{im} \varphi$.

Applying Lemma 3.1 to the lattice $N$ (see (3.2)), we see that there is a polarized K3 surface $(X, H)$ such that the Picard lattice of $X$ is $N$ with $H=\sum_{i=0}^{n} a_{i} \xi$. We set

$$
\begin{aligned}
v_{i} & :=r+\xi_{i}+a \rho_{X}, 0 \leq i \leq n, \\
v & :=\sum_{i=0}^{n} a_{i} v_{i} .
\end{aligned}
$$

Then we get that

$$
\left\{\begin{array}{l}
\left\langle v_{i}, v_{j}\right\rangle=-a_{i j} \\
\left\langle v, v_{j}\right\rangle=0 \\
\left\langle\widehat{H}, v_{j}\right\rangle=0
\end{array}\right.
$$

Let $E_{i}$ be a $v$-twisted semi-stable sheaf with $v\left(E_{i}\right)=v_{i}$ (Proposition 1.2). If $E_{i}$ is properly $v$-twisted semistable, then $\operatorname{rk} \operatorname{Pic}(X)>\operatorname{rk} N$, which is a contradiction. Hence $E_{i}$ is $v$-twisted stable for all $i$. Thus $\bar{M}_{H}^{v}(v)$ has a rational double point of type $\left(a_{i, j}\right)_{i, j=1}^{n}$. In particular, Remark 3.1 implies that there is a moduli space $\bar{M}_{H}^{v}(v)$ which has a rational double point of type $A_{n}, D_{n}, n \leq 18$, or $E_{n}$. 


\section{Appendix}

Finally we treat the wall crossing phenomenon under a wall $W_{u}$ with $u \in \mathcal{U} \backslash \mathcal{U}^{\prime}$. Assume that $\alpha$ belongs to exactly one wall $W_{u}$. Let $F$ be a $v+\alpha$-twisted semi-stable sheaf with $v(F)=u$. By our assumption, $F$ must be $v+\alpha$-twisted stable. We set $\alpha^{ \pm}:= \pm \epsilon v+\alpha$. We consider the Fourier-Mukai transform $\mathcal{F}_{\mathcal{E}}$ in (1.18). Then we see that $\operatorname{Ext}^{1}(F, E)=0$ for $E \in M_{H}^{v+\alpha}(v)$ : Indeed, if $\operatorname{Ext}^{1}(F, E) \neq 0$, then we have a non-trivial extension $0 \rightarrow E \rightarrow G \rightarrow F \rightarrow 0$ and we see that $G$ is a $v+\alpha$-twisted stable. On the other hand, $\left\langle v(G)^{2}\right\rangle<-2$, which is a contradiction. Hence $\operatorname{Ext}^{1}(F, E)=0$. Then, we have an isomorphism

$$
\begin{aligned}
M_{H}^{v+\alpha^{-}}(v) & \rightarrow M_{H}^{v+\alpha^{-}}\left(v^{\prime}\right) \\
H^{\prime} & \mapsto \mathcal{F}_{\mathcal{E}}^{1}(E)
\end{aligned}
$$

where $v^{\prime}=R_{v(F)}(v)$. We also have an isomorphsim

$$
\begin{array}{rlr}
M_{H}^{v+\alpha^{+}}\left(v^{\prime}\right) & \rightarrow & M_{H}^{v+\alpha^{+}}(v) \\
& \mapsto \mathcal{F}_{\mathcal{E}}^{1}(E)
\end{array}
$$

Since $\alpha$ belongs to exactly one wall, we get that $M_{H}^{v+\alpha^{-}}\left(v^{\prime}\right)=M_{H}^{v+\alpha}\left(v^{\prime}\right)=M_{H}^{v+\alpha^{+}}\left(v^{\prime}\right)$. Therefore we get an isomorphism $M_{H}^{v+\alpha^{-}}(v) \rightarrow M_{H}^{v+\alpha^{+}}(v)$ and under this identification, we get $\theta_{v}^{\alpha^{-}}=\theta_{v}^{\alpha^{+}}$.

\section{REFERENCES}

[A] Abe, T., A remark on the 2-dimensional moduli spaces of vector bundles on K3 surfaces, Math. Res. Lett. 7 (2000), 463-470

[E-G] Ellingsrud, G., Göttsche, L., Variation of moduli spaces and Donaldson invariants under change of polarization, J. Reine Angew. Math. 467 (1995), 1-49

[Li] Li, J., Compactification of moduli of vector bundles over algebraic surfaces, Collection of papers on geometry, analysis and mathematical physics, World Sci. Publishing, River Edge, NJ, (1997), 98-113

[M-W] Matsuki, K., Wentworth, R., Mumford-Thaddeus principle on the moduli space of vector bundles on an algebraic surface, Internat. J. Math. 8 (1997), 97-148

[Mu1] Mukai, S., Symplectic structure of the moduli space of sheaves on an abelian or K3 surface, Invent. math. 77 (1984), $101-116$

[Mu2] Mukai, S., On the moduli space of bundles on K3 surfaces I, Vector bundles on Algebraic Varieties, Oxford, 1987, $341-413$

[MFK] Mumford, D., Fogarty, J., Kirwan, F., Geometric invariant theory, Third edition. Ergebnisse der Mathematik und ihrer Grenzgebiete (2) 34. Springer-Verlag, Berlin, 1994.

[Na] Nakajima, H., Varieties associated with quivers, Representation theory of algebras and related topics (Mexico City, 1994), 139-157, CMS Conf. Proc., 19, Amer. Math. Soc., Providence, RI, 1996.

[S-N] Shimada, I., Zhang, D.-Q., Classification of extremal elliptic K3 surfaces and fundamental groups of open K3 surfaces, Nagoya Math. J. 161 (2001), 23-54

[T] Thaddeus, M., Stable pairs, linear systems and the Verlinde formula, Invent. Math. 117 (1994), 317-353

[Y1] Yoshioka, K., Some examples of Mukai's reflections on K3 surfaces, J. reine angew. Math. 515 (1999), 97-123

[Y2] Yoshioka, K., Moduli spaces of stable sheaves on abelian surfaces, math.AG/0009001, Math. Ann. 321 (2001), 817-884

[Y3] Yoshioka, K., Twisted stability and Fourier-Mukai transform I, Compositio Math. to appear

[Y4] Yoshioka, K., Twisted stability and Fourier-Mukai transform II. preprint

[Y5] Yoshioka, K., A note on Fourier-Mukai transform, math.AG/0112267

Department of mathematics, Faculty of Science, Kobe University, Kobe, 657, Japan

E-mail address: onishi@math.kobe-u.ac.jp, yoshioka@math.kobe-u.ac.jp 